
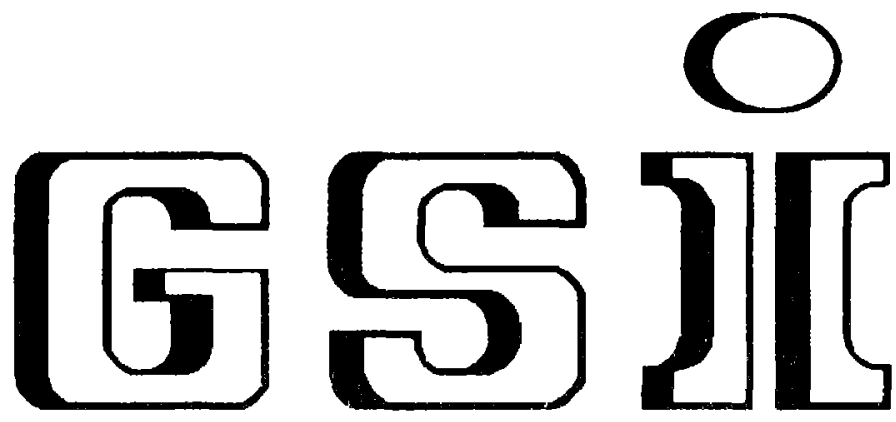

Preprint 2003 $-27+28$

August

2003-27 Experiment

The Electron-Electron Interaction Studied in Strong Central Fields by Resonant Transfer and Exitation with $\mathrm{H}$-like $\mathrm{U}$ lons

X. Ma, P.H. Mokler, F. Bosch, A. Gumberidze, C. Kozhuharov, D. Liesen,

D. Sierpowski, Z. Stachura, Th. Stölker, A. Warczak

2003-28 Theory

Angular Distribution of Hypersatellite and Satellite Radiation Emitted after Resonant and Excitation into $U^{91+}$ Ions

S. Zakowicz, Z. Harman, N. Grün, W. Scheid

(to be published in Phys. Rev. A)

Gesellschaft für Schwerionenforschung $\mathrm{mbH}$

Planckstraße 1 - D-64291 Darmstadt - Germany

Postfach 110552 - D-64220 Darmstadt • Germany 



\title{
The Electron-Electron Interaction Studied in Strong Central Fields by Resonant Transfer and Excitation with $\mathrm{H}$-like $\mathrm{U}$ Ions
}

\author{
X. Ma ${ }^{1,2}$, P.H. Mokler ${ }^{1}$, F. Bosch ${ }^{1}$, A. Gumberidze ${ }^{1}$, C. Kozhuharov ${ }^{1,}$ D. Liesen ${ }^{1}$, \\ D. Sierpowski ${ }^{3}$, Z. Stachura ${ }^{4}$, Th. Stöhlker ${ }^{1}$ and A. Warczak ${ }^{3}$ \\ ${ }^{I}$ GSI (Gesellschaft für Schwerlonenforschung), Darmstadt (Germany), \\ ${ }^{1,2}$ Institute of Modern Physics, 730000 Lanzhou (China), \\ ${ }^{3}$ Jagiellonian University, Institute of Physics, Krakow, Poland, \\ ${ }^{4}$ Institute of Nuclear Physics, Krakow, Poland

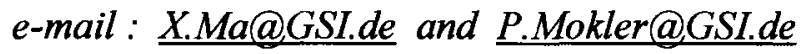

\begin{abstract}
Electron-electron interaction is studied in the strongest possible atomic fields $(Z \cdot \alpha \Rightarrow 1)$ in the presence of only two electrons. A quasi-free electron from a hydrogen gas target is resonantly captured into a $\mathrm{L}_{\mathrm{j}}$ subshell of a fast $\mathrm{H}$-like $\mathrm{U}^{91+}$ ion by simultaneous excitation of the strongly bound $\mathrm{K}$ electron also into a $\mathrm{L}_{\mathrm{j}}$, subshell of the projectile, with $j$ and $j$ ' the total angular momenta of $1 / 2$ or $3 / 2$ for the electron of concern. This resonant capture and excitation process, $\mathrm{KL}_{\mathrm{j}} \mathrm{L}_{\mathrm{j}}$-RTE, is mediated by electron-electron interaction. It is equivalent to dielectronic recombination (DR) in ionelectron collisions and leads to a doubly excited He-like $\mathrm{U}^{90+* *}$ ion, which stabilizes almost exclusively - via the emission of two successive $\mathrm{K} x$-rays, first a $\mathrm{K}$ hypersatellite $(\mathrm{K} \alpha \mathrm{i}-\mathrm{H})$ and then a $\mathrm{K}$ satellite $\left(\mathrm{K} \alpha \mathrm{i}^{\prime}-\mathrm{S}\right)$ transition. The $\mathrm{K}$ x-ray emission characteristics associated with one-electron capture in collisions of $\mathrm{U}^{91+}$ ions with a hydrogen target is studied for the three resonance groups of the $K L_{j} L_{j}$-RTE and one off-resonance energy, i.e. in the energy range between 100 and $135 \mathrm{MeV} / \mathrm{u}$. The total cross section for the first resonance group $\mathrm{KL}_{1 / 2} \mathrm{~L}_{1 / 2}$ confirms the importance of the Breit contribution to the interaction. The angular distribution for the $\mathrm{K} \alpha 2-\mathrm{H}$ transition $(\mathrm{j}=1 / 2)$ is isotropic in the projectile system whereas the $\mathrm{K} \alpha 1-\mathrm{H}$ transition $(\mathrm{j}=3 / 2)$ indicates a strong alignment for the $3 / 2$ electrons in the doubly excited states for the second resonance group $\mathrm{KL}_{1 / 2} \mathrm{~L}_{3 / 2}$. The experimental results are in agreement with fully relativistic calculations including the generalized Breit interaction.
\end{abstract}

PACS: $34.70 .+e, 32.30 . R j, 31.25 .-v$ 


\section{INTRODUCTION:}

Recombination processes, governed by electron-electron interaction - the so called dielectronic recombination "DR" - are of great importance in all plasmas from stellar origin to laser induced plasmas [1], for X-ray lasers [2] as well as for accelerator physics in particular with highly-charged heavy ions [3]. DR was first observed as mysterious dielectronic satellite lines in the solar optical spectrum and in 1964 explained theoretically by Burgess [4]. The first step of DR, the resonant electron capture, involves two electrons and forms a doubly excited ion, which subsequently stabilizes radiatively. The first part is just the time reversed Auger process mediated by the electron-electron interaction. In contrast to the Auger process, normally observed in singly ionized low- $Z$. atoms, DR reflects the electron-electron interaction for highly charged ions. Hence, DR has been extensively studied for heavy few-electron ions in recent years, see e.g. Ref. [5]. This new kind of research was especially also promoted by the novel possibilities arising with the advent of cooler electron targets at ion storage rings.

For heavy ionic systems the Breit interaction starts to play a dominant role [6-8]. In extreme cases, the reaction rates increase even by an order of magnitude compared to the pure Coulomb interaction [6]. Unfortunately, for heavy atomic systems the innermost electrons - the K electrons, which probe the strongest possible electric fields [9] - are presently difficult to access by DR measurements at cooler-storage rings. In order to excite a $\mathrm{K}$ electron of a heavy ion for instance to the $\mathrm{L}$ shell under resonant capture of a free electron (assuming also to the L shell), high electron energies with respect to the ion are needed. To date, such large detuning energies have not been provided at electron cooler targets. However, atomically confined quasi-free target electrons can provide the necessary kinetic energies for those $K \mathrm{~L}_{\mathrm{j}} \mathrm{L}_{\mathrm{j}}$, resonances. Here, we use the Auger notation with $\mathrm{j}$ and $\mathrm{j}$ ' the total angular momenta of the electrons in the created doubly excited state. The doubly excited ion can stabilize radiatively under the emission of the corresponding $\mathrm{K}$ x-rays, which is the second step in this resonant transfer and excitation process, the $\mathrm{KL}_{\mathrm{j}} \mathrm{L}_{\mathrm{j}}$-RTE. Compared to $\mathrm{DR}$ involving free electrons, which are magnetically confined by the cooler solenoid, the RTE resonances are smeared out by the Compton profile of the atomically confined electrons. For very heavy ions the radiative stabilization is so fast that it dominates the normally competing Auger decay. Recently progress towards direct KLL-DR measurements have been made at the ESR storage ring using stochastic ion beam cooling and the "cooler" as pure electron target [10]. However, due to geometrical restrictions at cooler targets the photon emission, in particular its emission characteristics, is not accessible in DR measurements.

For very heavy projectiles, first measurements on $K L_{j} L_{j}$-RTE were reported from the BEVALAC in Berkeley for He-like $\mathrm{U}^{90+}$ ions colliding with electrons initially confined in $\mathrm{C}$ atoms [11] and then - more successfully - in $\mathrm{H}_{2}$ molecules [12] by measuring just the excitation function for the charge exchange cross sections. Due to the large spin-orbit splitting three resonance groups for the three different combinations of the total angular momenta $\mathrm{j}$ and $\mathrm{j}$ ' (with $1 / 2$ or $3 / 2$ ) in the $\mathrm{K}$-shell excited Li-like $\mathrm{U}^{89+}$ could be resolved for the first time by RTE measurements. For lighter ions the three $K L_{j} L_{j}$-RTE subgroups merge together into only one broad KLL resonance group, cf. e.g. [13]. Differential investigations with the heaviest ion - He-like $\mathrm{U}^{90+}$ - were performed later at SIS in 
Darmstadt measuring the charge exchange in coincidence with the radiative stabilization, i.e. with the characteristic $\mathrm{K} x$-ray emission [14-16]. The $\mathrm{x}$-ray transitions associated with the different $\mathrm{j}$ levels could be resolved giving access to the different total angular momenta involved in the K-shell excited Li-like $\mathrm{U}^{89+*}$ states. Moreover, the measured emission characteristics gave access to the alignment of the excited states [17]. In accordance with fully relativistic calculations [18], the Breit term will enhance the cross section for the first resonance group $\left(\mathrm{KL}_{1 / 2} \mathrm{~L}_{1 / 2}\right)$ roughly by an overall factor of two. Moreover, the Breit interaction influences the magnetic substate population; in the second resonance group $\left(\mathrm{KL}_{1 / 2} \mathrm{~L}_{3 / 2}\right)$ electrons with $\mathrm{j}=3 / 2$ are strongly aligned perpendicular to the ion flight direction. Theoretical studies on the alignment of doubly excited states created by DR (or RTE) show a considerable variation over atomic systems. In particular, the alignment varies with the strength of the probed central field $[18,19]$.

For the first time we report here in detail on a measurement of the heaviest available, most simple accessible atomic system where an electron-electron interaction can occur: a one-electron (H-like) $\mathrm{U}^{91+}$ ion captures by resonant excitation a quasi-free target electron atomically confined in a $\mathrm{H}_{2}$ molecule [20]. In this $\mathrm{KL}_{\mathrm{j}} \mathrm{L}_{\mathrm{j}}$ - $-\mathrm{RTE}$ process only two electrons are involved and the $\mathrm{H}_{2}$ gas target used provides the narrowest Compton profile available. Once more, the RTE process is determined via coincidences between the stabilizing characteristic $\mathrm{X}$-ray emission and the associated single electron capture events. Moreover the complete $\mathrm{x}$-ray emission pattern is measured in order to extract information on the alignment of the intermediately formed doubly excited states of He-like $U^{90+} * *$. In contrast to Li-like ions with a single K-shell excitation as we have it in $\mathrm{U}^{89+}$ ions, for doubly exited He-like ions as $\mathrm{U}^{90+* *}$ two $\mathrm{K}$ vacancies exist with the subsequent emission

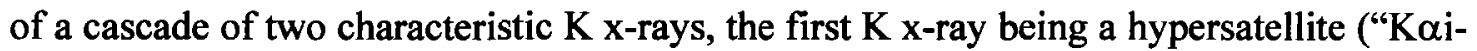
$H$ ") transition and the second one a satellite ("Kai'-S") transition $\left(i, i^{\prime}=1,2\right)$. Due to the screening of the nuclear charge by the first electron filling the $\mathrm{K}$ shell, the second transition - the "K$\alpha i$ '-S" transition - has a slightly lower transition energy than the first

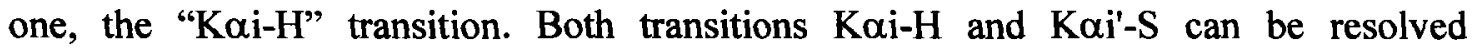
energetically with conventional solid-state $\mathrm{x}$-ray detectors. The advantage of observing $\mathrm{K} \alpha \mathrm{i}-\mathrm{H}$ transitions is that the competing radiative electron capture process, $\mathrm{REC}-$ see e.g. [21], cannot produce hypersatellites in the initially $\mathrm{H}$-like ions. The $\mathrm{K} \mathrm{x}$-ray cascades

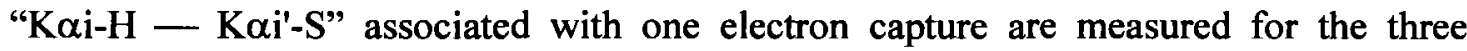
$\mathrm{KL}_{\mathrm{j}} \mathrm{L}_{\mathrm{j}}$ '-RTE resonance groups (1-R, 2-R, 3-R corresponding to $\mathrm{KL}_{1 / 2} \mathrm{~L}_{1 / 2}-\mathrm{KL}_{1 / 2} \mathrm{~L}_{3 / 2^{-}}$, $\left.\mathrm{KL}_{3 / 2} \mathrm{~L}_{3 / 2}-\mathrm{RTE}\right)$ and one off-resonance energy (O-R) in the energy range between 100 and $135 \mathrm{MeV} / \mathrm{u}$. In the accompanying paper by Zakowicz et al. [22] a fully relativistic description of the RTE processes relevant to this experiment is given and the calculations are compared with the experimental results presented here.

\section{FORMATION AND DECAY OF DOUBLY EXCITED $U^{90+* *}$}

The resonant formation of a doubly excited state and its subsequent radiative stabilization is the fingerprint of the RTE process. For the three possible resonance groups in $\mathrm{KL}_{\mathrm{j}} \mathrm{L}_{\mathrm{j}}$ '$\mathrm{RTE}$ the formation and the radiative stabilization by a $\mathrm{K} \alpha \mathrm{i}-\mathrm{H}-\mathrm{K} \alpha \mathrm{i}^{\prime}-\mathrm{S} \mathrm{x}$-ray cascade is exemplified in Fig.1 (a)-(c). On the left side in each case the formation of the doubly

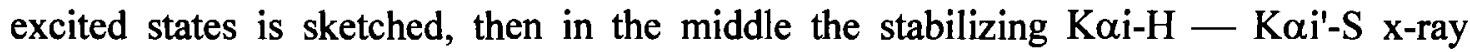


cascade, and for comparison on the right side the competing REC process. Fig.1-(a) gives the case for $K L_{1 / 2} L_{1 / 2}-R T E$, first resonance group "1-R": Only $\mathrm{K} \alpha 2-\mathrm{H}-\mathrm{K} \alpha 2-\mathrm{S}$ radiation can be emitted, i.e. only transitions originating from a $\mathrm{j}=1 / 2 \mathrm{~L}$ sublevel, the $\mathrm{K} \alpha 2-\mathrm{H}$ and $\mathrm{K} \alpha 2-\mathrm{S}$ lines, can be observed. K$\alpha 2-\mathrm{S}$ transitions can also be caused by REC to the $L_{1 / 2}$ subshell and its cascade down to the $K$ shell. Similarly REC to the $L_{3 / 2}$ level is possibly producing in the end a $K \alpha 1-S$ cascade line ( $\mathrm{L}_{3 / 2}$ to $\mathrm{K}$ transition). A hypersatellite line cannot be produced by REC. However, for the considered resonance case the centroid energy of the broad $\mathrm{L}_{1 / 2}-\mathrm{REC} \mathrm{x}$-ray distribution coincides with the $\mathrm{K} \alpha 2-\mathrm{H}$ line. Fortunately, both the contributions can be separated via the huge difference in their widths, assuming no interference between RTE and REC. The narrow $\mathrm{K} \alpha 2-\mathrm{H}$ line will stick out of the broad $\mathrm{L}_{1 / 2}-\mathrm{REC} \mathrm{x}$-ray distribution (cf. Fig. 4 below).

Similar arguments hold true for the second RTE group " $2-\mathrm{R}$ ", the $\mathrm{KL}_{1 / 2} \mathrm{~L}_{3 / 2}$ resonances. From the RTE process, levels with both $\mathrm{j}$ values, $1 / 2$ and $3 / 2$, are populated simultaneously - see Fig.1-(b). Consequently, the first K x-ray can be either a Ka2-H or a $\mathrm{K} \alpha 1-\mathrm{H}$ transition (starting with a $1 / 2$ or $3 / 2 \mathrm{~L}$ electron) and the second $\mathrm{K} \mathrm{x}$-ray is then a

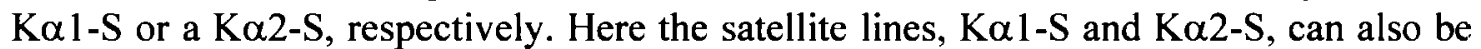
produced as cascade lines from $\mathrm{L}_{3 / 2}$ and $\mathrm{L}_{1 / 2}-\mathrm{REC}$, respectively. In the same way we find underneath the sharp hypersatellite lines, $\mathrm{K} \alpha 2-\mathrm{H}$ and $\mathrm{K} \alpha 1-\mathrm{H}$, the corresponding broad $\mathrm{L}_{3 / 2^{-}}$and $\mathrm{L}_{1 / 2}$ - REC $\mathrm{x}$-ray distributions.

For the third resonance group, " $3-\mathrm{R}$ " corresponding to $\mathrm{KL}_{3 / 2} \mathrm{~L}_{3 / 2}-\mathrm{RTE}$, both the electrons in the doubly excited states are in $\mathrm{j}=3 / 2$ levels. Consequently we observe due to RTE only the $\mathrm{K} \alpha 1-\mathrm{H}-\mathrm{K} \alpha \mathrm{l}-\mathrm{S}$ cascade. The competing $\mathrm{L}_{3 / 2^{-}} \mathrm{REC}$ will contribute via the cascade to the $\mathrm{K} \alpha \mathrm{l}-\mathrm{S}$ transitions and the broad $\mathrm{L}_{3 / 2}$ - REC $\mathrm{x}$-ray distribution will lie under the sharp K $\alpha 1-H$ line of the RTE process. Obviously, we will also observe the $L_{1 / 2}-R E C$ and its $\mathrm{K} \alpha 2-\mathrm{S}$ cascade line.

Naturally, the $\mathrm{x}$-ray emission associated with the REC process can be studied undisturbed from RTE at an off-resonance energy "O-R". Assuming no interference between RTE. and REC, the REC contributions found at O-R can be used to disentangle RTE and REC contributions at the different resonance groups. For the measurements described below with initial $\mathrm{H}$-like $\mathrm{U}^{91+}$ ions, we used energies of $102.0 \mathrm{MeV} / \mathrm{u}$ for $\mathrm{O}-\mathrm{R}, 116.6 \mathrm{MeV} / \mathrm{u}$ for 1-R, $124.9 \mathrm{MeV} / \mathrm{u}$ for $2-\mathrm{R}$, and $133.1 \mathrm{MeV} / \mathrm{u}$ for 3-R. The resonance energies were calculated using the total binding energies for the corresponding resonance groups with the help of the GRASP structure program [23] and were taken from Ref. [24].

\section{EXPERIMENTAL ARRANGEMENT}

The experiment was performed at the heavy ion accelerator facility of GSI in Darmstadt. $U$ ions were accelerated by the heavy ion synchrotron SIS to specific energies of about $280 \mathrm{MeV} / \mathrm{u}$. After stripping in a copper foil, H-like ions were injected into the storage ring ESR, cooled there by merging a cold electron beam, and then decelerated in the ESR down to the required energies $(102.0,116.6,124.9$, and 133.1 MeV/u). At the measuring energies, the ion beam was energetically fixed and cooled again by electron cooling. By cooling the beam diameter shrank, and the ion beam with its diameter of about $2 \mathrm{~mm}$ intersected an $\mathrm{H}_{2}$ gas jet target. The $\mathrm{H}_{2}$ gas and the nozzle of the jet target were precooled to liquid nitrogen temperature in order to achieve high target densities through 
cluster formation. The areal densities ranged up to about $10^{12} \mathrm{H} / \mathrm{cm}^{2}$. For details of the gas jet arrangement see [25].

$\mathrm{U}^{90+}$ ions - having captured one electron in the gas jet target - were separated from the circulating $\mathrm{U}^{91+}$ ions in the next down-stream bending magnet of the ESR and detected behind it in a particle detector inserted into a moveable pocket with a thin $(50 \mu \mathrm{m})$ stainless steel window [26]. For the general layout of the arrangement see Fig.2. For particle detection a position sensitive fast gas counter was used. Around the gas jet a photon detection chamber is installed giving access to observation angles in the laboratory frame near $10^{\circ}$ and of $35^{\circ}, 60^{\circ}, 90^{\circ}, 120^{\circ}$, and $150^{\circ}$; for details see [27]. The photon detection chamber is equipped for x-ray observation with thin $50 \mu \mathrm{m}$ stainless steel and $100 \mu \mathrm{m}$ Be windows at forward and backward observation angles, respectively. The x-ray detection chamber is inserted with an enlarged scale in the center of Fig.2. For $\mathrm{x}$-ray detection, standard solid state Ge(i) detectors were used with collimators in front of them in order to reduce the angular energy dispersion caused by the Doppler effect. The $10^{\circ}$ detector was a special one, segmented into four parallel stripes looking sideward and giving access to four adjacent angles - only two of them were used during the experiment; here, no collimation was needed. Solid angles were calculated from the geometry of the arrangement, and detector efficiencies were determined using calibrated radioactive sources. In Table 1, detector size, slit geometry, solid angle, window material and thickness, detector efficiency and resolution are given for the different observation angles. The detector efficiencies refer to the typical $100 \mathrm{keV}$ radiation in the ion frame where an ion energy of $116.6 \mathrm{MeV} / \mathrm{u}$ was assumed here; the corresponding Lab energies are given in the brackets for completeness.

The emitted $x$ rays were recorded in coincidence with the down-charged $U^{90+}$ ions in event mode using standard NIM and CAMAC modules for signal processing and GSIMBS data acquisition system. In Fig.3, typical raw coincident $x$-ray spectra taken at $150^{\circ}$ for the four measuring energies ( $O-R, 1-R, 2-R$, and 3-R) are shown in a 3-D representation. For convenience, the $\mathrm{x}$-ray energies are already transformed into the emitter frame of the ion. The identifications of the lines are sketched in the projection plane at the top of the figure. For the off-resonance case (O-R), we see only the broad $\mathrm{L}_{3 / 2}$ - and $\mathrm{L}_{1 / 2}-\mathrm{REC}$ lines (around 85.0 and $89.4 \mathrm{keV}$ ) and the sharp cascade contributions $\mathrm{K} \alpha 2-\mathrm{S}$ and $\mathrm{K} \alpha 1-\mathrm{S}$ (at about 96.2 and $100.6 \mathrm{keV}$ ). According to the increase in ion energy the broad L-REC lines shift successively to higher $x$-ray energies for the resonance groups 1-R, 2-R, and 3-R, respectively. At 1-R the $\mathrm{L}_{1 / 2}$ - REC line coincides with the new narrow $\mathrm{K} \alpha 2-\mathrm{H}$ line (at $97.5 \mathrm{keV}$ ) caused by $\mathrm{RTE}$; at 2-R the $\mathrm{L}_{1 / 2}-\mathrm{REC}$ line coincides already with the new $\mathrm{K} \alpha 1-\mathrm{H}$ line (at $101.9 \mathrm{keV}$ ) from RTE and the $\mathrm{L}_{3 / 2}-\mathrm{REC}$ with the $\mathrm{K} \alpha 2-\mathrm{H}$ line from RTE. And finally, at 3-R there is no $\mathrm{K} \alpha 2-\mathrm{H}$ line, the $\mathrm{L}_{3 / 2}-\mathrm{REC}$ coincides with the $\mathrm{K} \alpha 1-\mathrm{H}$ from RTE and the $\mathrm{L}_{1 / 2}$ - REC contribution is already outside the region of interest. For the two high ion energy spectra at 2-R and 3-R, the REC contributions to the $M$ shell show up at the low $x$-ray energy side.

At the other observation angles the spectra look quite similar. However, due to the strong variation of the angular distribution for the broad REC radiation [28] the visibility of the $\mathrm{K} \alpha \mathrm{i}-\mathrm{H}$ lines changes with angle. The REC contributions are especially strong around observation angles of $90^{\circ}$. 


\section{DECOMPOSITION OF SPECTRA}

The $28 \mathrm{x}$-ray spectra taken at the different ion energies and at all the observation angles were decomposed into the various contributions using Gaussian distributions for the lines and a linear background subtraction. The line widths for the broad REC lines as well as their intensity ratio were fixed according to the expectations whenever it was necessary for the fitting routine. Here, the spectra at O-R give a good starting value. Different fitting routines were used applying also standard response line shapes for the lines of the $\mathrm{K} \mathrm{x}$-ray transitions and appropriately broader distributions for the REC contributions. At the statistical level available, however, no real variance in the result could be observed. So we stick in the following to the decomposition of the spectra using Gaussian line distributions. The negligible variance in the different methods for spectra deconvolution gives us confidence on small systematic errors of our analysis.

Examples for the decomposition of the spectra are given in Fig.4 for the four ion energies at $\mathrm{O}-\mathrm{R}, 1-\mathrm{R}, 2-\mathrm{R}$, and $3-\mathrm{R}$ for one observation angle of $14.7^{\circ}$. In the spectra the true counts per channel are given in order to be able to judge on statistics. Due to the Doppler effect the lines shift in channel for the different ion energies; for convenience in the representation we adjust the spectra in such a way that the main characteristic $\mathrm{K}$ lines stay at the same position. In the semi-logarithmic plot the different contributions are clearly visible. As mentioned already the L- REC lines shift with increasing ion energy to higher $\mathrm{x}$-ray energies: at $1-\mathrm{R}$ the broad $\mathrm{L}_{1 / 2}-\mathrm{REC}$ distribution is right under the narrow $\mathrm{K} \alpha 2-\mathrm{H}$ line, at $2-\mathrm{R}$ both the $\mathrm{L}_{\mathrm{j}}-\mathrm{REC}$ contributions are under both the hypersatellite lines $\mathrm{K} \alpha \mathrm{i}-\mathrm{H}$, at 3-R finally the $\mathrm{L}_{3 / 2}-\mathrm{REC}$ part shifts under the $\mathrm{K} \alpha 1-\mathrm{H}$ line.

From the decomposition of the spectra we obtain relative line intensities for each spectrum. These relative line intensities are summarized in Table $\mathbf{2}$ for all the observation angles and all the ion energies used. For convenience to the reader detector efficiencies (cf. Table 1) have already been incorporated into the numbers given. Hence, after incorporating the solid angles the intensities for one ion energy will give the relative angular differential cross sections in the laboratory system. Additionally these values still have to be normalized to the reaction product of the number of ions times the target density and measuring time. In order to get absolute cross sections, we have to normalize the $\mathrm{K} \alpha$ line intensities to the $\mathrm{K}-\mathrm{REC}$ line that shows up at higher $\mathrm{x}$-ray energies. The $\mathrm{K}$ REC radiation was separately registered in coincidence with capture events using a second set of main amplifiers with lower amplification. Additionally, the K- REC intensities - also corrected for efficiency - are given in Table 2. By applying this normalization procedure, all the systematic errors are canceled in the cross section calculations. The K- REC radiation is preferentially emitted perpendicular to the ion beam direction $[29,30]$, and its angular distribution has to be taken into account. On the other hand normalizing the $90^{\circ}$ data to the K- REC is sufficient if for the other angles the solid angle corrections are included into the numbers given in Table 2. For completeness we add in the last column the theoretical angular differential cross sections for K- REC per $\mathrm{K}$ vacancy in $\mathrm{U}^{92+}$ as calculated by Eichler et al. [31, 32]. 


\section{EXPERIMENTAL RESULTS AND DISCUSSIONS}

As the $\mathrm{K} \alpha 2-\mathrm{S}$ line is a 1/2-to-1/2 transition, on a first glance we may expect in the emitter frame an isotropic emission pattern. However, in reality we have to consider the total angular momenta involved for the relevant two-electron states [22]. In Fig. 5 the angular differential $\mathrm{K} \alpha 2-\mathrm{S} \mathrm{x}$-ray emission cross sections as observed in the laboratory system $\left(d \sigma_{\mathrm{K} \alpha 2-\mathrm{S}} / \mathrm{d} \Omega_{\mathrm{LAB}}\right)$ are plotted for the off-resonance case $(\mathrm{O}-\mathrm{R})$. Due to the Lorentz transformation the emission in the laboratory system is strongly forward peaked and coincides perfectly with an isotropic emission pattern in the moving ion system (emitter system) transformed into the laboratory frame. In the lower part of the figure the ratio of the data to the expectation of the Lorentz transformation (K $\alpha 2-\mathrm{S} /$ Lorentz) is plotted, confirming the isotropic emission characteristic.

Also for the three resonance energies, 1-R, 2-R, and 3-R, the emission patterns for the $\mathrm{K} \alpha 2-\mathrm{S}$ lines are experimentally found to be practically isotropic in the emitter frame of

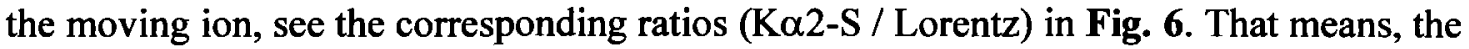
$\mathrm{K} \alpha 2-\mathrm{S}$ lines display experimentally an isotropic emission independent of the ion energies and possibly also independent of the production process - i.e. cascades from RTE or from REC. Considering the strong REC cascade contribution to the $\mathrm{K} \alpha 2-\mathrm{S}$ line this does not necessarily mean that also all the RTE contributions have to be isotropic: A small anisotropic RTE contribution may not disturb the observed isotropy at the available level of experimental significance (compare Ref. [22]). As the total $\mathrm{K} \alpha 2-\mathrm{S}$ emission is observed to be isotropic, we will use this fact to normalize all our other lines to the K $\alpha 2$ $\mathrm{S}$ line. Hence, considering the corresponding line intensity ratios all solid angle corrections and transformation factors will cancel and the ratios will just give the emission patterns in the emitter frame (however displayed for the actual observation angles in the laboratory).

The $\mathrm{K} \alpha 1-\mathrm{S}$ line is a 3/2-to-1/2 transition and its emission characteristics may depend on its production mechanisms. In Fig. 7 the ratios $\mathrm{K} \alpha 1-\mathrm{S} / \mathrm{K} \alpha 2-\mathrm{S}$ are plotted for all the measured ion energies, $\mathrm{O}-\mathrm{R}, 1-\mathrm{R}, 2-\mathrm{R}$, and 3-R. For all ion energies only negligible anisotropies are observed. However, within the measuring accuracies in all the cases also the $\mathrm{K} \alpha 1-\mathrm{S}$ emission can be considered as isotropic. The average values for the Kal-S / $\mathrm{K} \alpha 2-\mathrm{S}$ ratios are about $0.435 \pm 0.030$ at $\mathrm{O}-\mathrm{R}, 0.340 \pm 0.018$ at $1-\mathrm{R}, 0.466 \pm 0.016$ at $2-\mathrm{R}$, and $0.467 \pm 0.032$ at $3-R$. The $O-R$ value is only caused by $R E C$ contributions, whereas the ratios at the different resonances in comparison to the off-resonance value give a crude number for the additional relative cascade contributions to the satellite $\mathrm{x}$-ray emission caused by RTE, which will be considered below.

However, first we like to emphasize that for bare projectiles a strong anisotropy was observed for the K $\alpha 1-\mathrm{S}$ emission from pure REC cascades [30] pointing there to a strong alignment for the captured electron. This is in evident contrast to our finding for the $\mathrm{H}$ like projectiles. In how far here a coupling to the second electron - we have intermediate two-electron states - influences the emission pattern will be discussed elsewhere.

In contrast to O-R where only REC cascades contribute to the $\mathrm{K} \alpha 1-\mathrm{S} / \mathrm{K} \alpha 2-\mathrm{S}$ emission, at 1-R additionally RTE contributes also to the $\mathrm{K} \alpha 2-\mathrm{S}$ line and the $\mathrm{K} \alpha 1-\mathrm{S} / \mathrm{K} \alpha 2-\mathrm{S}$ ratio decreases correspondingly. From the experimental value of 0.340 we can extract that the RTE cascade contribution to the K $\alpha 2-\mathrm{S}$ transition is only a fraction of about 0.28 of the 
REC contribution. Or referring to the total line intensity, only about $22 \%(=1 / 4.58$, see below) of the Ka2-S line is caused by RTE. This evaluation assumes that the Ka1-S / $\mathrm{K} \alpha 2-\mathrm{S}$ ratio caused by REC cascades alone does not change with ion energy.

For the other resonance groups, especially for $2-R$, it is difficult to extract from the experimental ratios $\mathrm{K} \alpha 1-\mathrm{S} / \mathrm{K} \alpha 2-\mathrm{S}$ directly the RTE cascade contributions to these lines. However, we can use as additional input the calculated ratios of the RTE cross sections for the different $x$-ray. Hence, we summarize in Table 3 the corresponding theoretical $x$ ray emission cross sections induced by RTE from ref. [22]. Here, the small contributions to $\mathrm{K} \alpha \mathrm{l}-\mathrm{HS}$ as well as to $\mathrm{K} \alpha 1-\mathrm{S}$ and to $\mathrm{K} \alpha 2-\mathrm{HS}$ as well as to $\mathrm{K} \alpha 2-\mathrm{S}$ in resonance group 1 and 3 respectively, are due to the overlap from the neighboring resonance groups induced by the Compton profile.

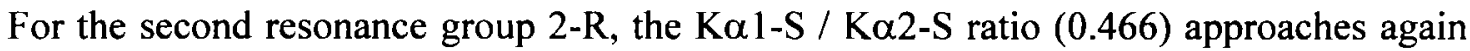
the off-resonance value (0.435), and overshoots it. This indicates that RTE contributes

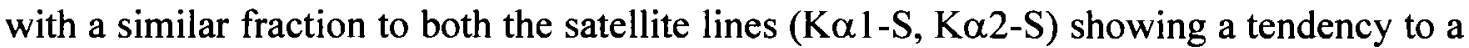

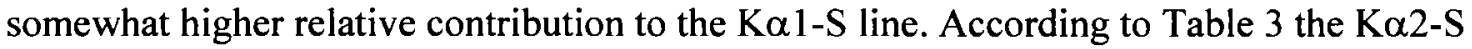
emission cross section for the RTE part at 2-R is only a fraction of 0.41 of that at $1-R$. A similar cross section ratio at both resonances was found experimentally also for the case of initially He-like $\mathrm{U}$ ions [14 - 16]. Assuming for the $\mathrm{K} \alpha 2-\mathrm{S}$ line at 2-R a RTE cascade contribution equivalent to the one at $1-\mathrm{R}$ reduced by the corresponding RTE cross section ratio, we find that only about $10.3 \%(=1 / 9.74)$ of the Ka2-S radiation is caused by $\mathrm{RTE}$. For the $\mathrm{K} \alpha \mathrm{l}-\mathrm{S}$ line we find a relative higher RTE cascade contribution of about 16 $\%$. (Nevertheless, the absolute emission cross section for $\mathrm{K} \alpha 1-\mathrm{S}$ is considerably smaller than that for K $\alpha 2-S$.) Due to the experimental uncertainties all these numbers can only be guidelines.

For the third resonance group 3-R, the $\mathrm{K} \alpha 1-\mathrm{S} / \mathrm{K} \alpha 2-\mathrm{S}$ ratio does not change further within the errors; an RTE cascade contribution to the $\mathrm{K} \alpha 2-\mathrm{S}$ line is not possible here. From this we deduce that RTE contributes only with a small part of about $7 \%$ to the $\mathrm{K} \alpha \mathrm{l}-\mathrm{S}$ emission. (For comparison this would corresponds to only about $3 \%$ of the K $\alpha 2$ $\mathrm{S}$ intensity). We emphasize that the RTE resonance strength at 3-R is relatively weak, $\mathrm{cf}$. Table 3.

Hypersatellite lines cannot be caused by REC cascade contributions at all. Hence, these lines give directly the contributions from the RTE process alone. As explained already, for $1-\mathrm{R}$ and $3-\mathrm{R}$ we expect contributions to the $\mathrm{K} \alpha 2-\mathrm{H}$ and $\mathrm{K} \alpha 1-\mathrm{H}$ lines respectively, and for $2-\mathrm{R}$ both the $\mathrm{K} \alpha 2-\mathrm{H}$ and $\mathrm{K} \alpha 1-\mathrm{H}$ lines. The corresponding ratios $\mathrm{K} \alpha 2-\mathrm{H} / \mathrm{K} \alpha 2-\mathrm{S}$ and $\mathrm{K} \alpha 1-\mathrm{H} / \mathrm{K} \alpha 2-\mathrm{S}$ are shown in Figs. 8 and 9, respectively. The $\mathrm{K} \alpha 2-\mathrm{H}$ is a $1 / 2-$ to- $1 / 2$ transition and it is not surprising that the $\mathrm{K} \alpha 2-\mathrm{H} / \mathrm{K} \alpha 2-\mathrm{S}$ ratios display isotropic emission patterns. Theory predicts for $\mathrm{K} \alpha 2-\mathrm{H}$ transitions also an isotropic emission [22]. The experimental mean absolute values for the $\mathrm{K} \alpha 2-\mathrm{H} / \mathrm{K} \alpha 2-\mathrm{S}$ ratios are about $0.24 \pm 0.03$ at $1-\mathrm{R}$ and $0.064 \pm 0.016$ at $2-\mathrm{R}$. The theoretical ratios calculated from the values in Table 3 are 1.00 and 0.48 , respectively. However, the theoretical values do not include the REC cascade contributions and, hence, have to be divided by the reduction factors quoted above of 4.58 and 9.74 yielding to $\mathrm{K} \alpha 2-\mathrm{H} / \mathrm{K} \alpha 2-\mathrm{S}$ ratios of 0.21 at $1-\mathrm{R}$ and 0.05 at $2-\mathrm{R}$. These theoretical predictions for the ratios agree quite nicely within the expected accuracies with the experimental findings (see also Fig 8). 
The $\mathrm{K} \alpha 1-\mathrm{H}$ emission corresponds to transitions from 3/2-to-1/2 states and may show anisotropic behavior due to the alignment caused by the RTE process. Indeed the K $\alpha 1-\mathrm{H}$ / $\mathrm{K} \alpha 2-\mathrm{S}$ ratio for the second resonance group 2-R shows a pronounced dipole emission pattern in the emitter frame, see Fig. 9. (At these ion energies a lab angle of $60^{\circ}$ corresponds roughly to $90^{\circ}$ in the emitter system.) From Table 3 we deduce an average $\mathrm{K} \alpha 1-\mathrm{H} / \mathrm{K} \alpha 2-\mathrm{S}$ ratio of 0.90 for RTE alone which has once more to be reduced by a factor 9.74 yielding a mean ratio of 0.093 . This is in good agreement with a mean experimental value around 0.1 . Moreover the theoretical expectation for the emission pattern included in Fig. 9 is also in reasonable agreement with the experimental findings. (In the theoretical ratio given here, we neglected the K $\alpha 2-\mathrm{S}$ contributions from RTE, only the K $\alpha 2-\mathrm{S}$ intensity caused by REC are used.)

For the resonance group 3-R the $\mathrm{K} \alpha 1-\mathrm{H} / \mathrm{K} \alpha 2-\mathrm{S}$ ratio shows an experimental value only around 0.04 which has to do with the fact that this resonance strength is pretty small and the REC contributions are correspondingly dominant. The emission pattern seems to be isotropic with a tendency of an opposite emission characteristics, possibly pointing to a reversed alignment compared to the second resonance 2-R. Considering only the RTE

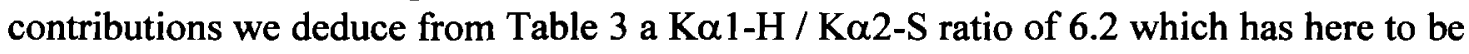
reduced roughly by a factor of 30 including REC contributions to the total $\mathrm{K} \alpha 2-\mathrm{S}$ emission. However, this ratio is totally blurred by a huge systematical uncertainty.

As REC does not contribute to the hypersatellite lines at all, for the second resonance group 2-R we can also consider the intensity ratio of the hypersatellites $K \alpha 1-\mathrm{H} / \mathrm{K} \alpha 2-\mathrm{H}$, which cannot be disturbed by any REC contributions. Since we showed (in Fig. 8) that the $\mathrm{K} \alpha 2-\mathrm{H}$ line displays an isotropic emission pattern, the $\mathrm{K} \alpha 1-\mathrm{H} / \mathrm{K} \alpha 2-\mathrm{H}$ ratio depicted in Fig. 10 should give directly the emission characteristic of the $\mathrm{K \alpha l}-\mathrm{H}$ line. This ratio can be compared to theory [22] on an absolute scale, as no reduction factors have to be applied. This absolute comparison (see full line in Fig. 10) demonstrates an overall good agreement. However, the experimental points may suggest a stronger anisotropy than predicted (see dotted line). This observed stronger anisotropy might be caused by contributions from higher multipole transitions, as in the theoretical treatment only electric dipole transitions have been included. A similar enhancement of the anisotropy caused by higher multipole contributions has been reported for the case of REC cascades for initially bare $\mathrm{U}^{92+}$ projectiles [33].

From the $\mathrm{K} \alpha 1-\mathrm{H} / \mathrm{K} \alpha 2-\mathrm{H}$ ratios we can extract an anisotropy parameter of $\boldsymbol{\beta}_{A}$ using

$$
\mathrm{W}(\theta) \propto 1+\boldsymbol{\beta}_{A} \cdot \mathrm{P}_{\mathbf{2}}(\cos \theta)
$$

(with $P_{2}$ the Legendre polynomial). A fit to the data leads to a value of $\boldsymbol{\beta}_{A}=-0.75$ (see dotted line in Fig. 10). In one-electron approximation (H-like System), $\boldsymbol{\beta}_{\boldsymbol{A}}$ can only vary between 0.5 and -0.5 for a single electron transition from $3 / 2$ to $1 / 2$ with dipole emission. Couplings to the second electron and possible higher multipole contributions will change this, cf. also [34]. In the accompanying publication [22] electron-electron coupling is already included into the calculations, however no higher multipole contributions are considered. Assuming for the moment the one-electron approximation, i.e. no coupling of the $j=3 / 2$ electron to the other one with $j=1 / 2$ and additionally no higher multipole contributions, the alignment $\boldsymbol{A}$ of the magnetic substate population $\boldsymbol{P}_{|\mathrm{mj}|}$ (with $\left|\mathrm{m}_{\mathrm{j}}\right|=1 / 2$ and $3 / 2$ ) would be given by

$$
\boldsymbol{A}=\left(\boldsymbol{P}_{|3 / 2|}-\boldsymbol{P}_{|1 / 2|}\right) /\left(\boldsymbol{P}_{|3 / 2|}+\boldsymbol{P}_{|1 / 2|}\right) \text {. }
$$


For such a dipole emission we have $\boldsymbol{\beta}_{A}=0.5 \cdot \boldsymbol{A}$, cf. [16]. With $\boldsymbol{\beta}_{A}=-0.5$ this yields to a value for the alignment of $\boldsymbol{A}=-1.0$, which means $\left|\mathrm{m}_{\mathrm{j}}\right|=1 / 2$ substates are preferentially populated in the $3 / 2$ level. Or in other words, only angular momenta $j$ perpendicular to the collision direction can be transferred for the $j=3 / 2$ states considered. It is interesting to note that for the third resonance group $3-\mathrm{R}$ where both the electrons are in $\mathrm{j}=3 / 2$ intermediate states the alignment $\boldsymbol{A}$ is zero or even slightly positive (cf. Fig. 9). This may mean that the Pauli exclusion principle in connection with the $\mathrm{j}-\mathrm{j}$ coupling destroys completely the alignment.

Finally, with the measured emission characteristics for the different lines we can determine the total strengths of the resonances by integrating over all the angles. For absolute normalization the K-REC cross sections calculated by Eichler and Ichihara [32] were used. The total RTE cross sections extracted from the hypersatellite contributions are summarized in Table 4 and compared to the theoretical values determined by the fully relativistic theory [22]. For comparison, also the cross sections not including the relativistic Breit term are given in brackets. For the first resonance group $1-R$ an appreciable difference between fully relativistic and non-relativistic calculations has been reported. The experimental values confirm evidently the fully relativistic theory including the complete Breit term for the interaction.

In the data analysis and interpretation given above, we did not use calculated L-REC cross sections in order to avoid any ambiguities. However, we like to stress that the predictions for the total L-REC cross sections from [32] agree well with the observed values. In particular, at $\mathrm{O}-\mathrm{R}$ the measured intensity ratio $\mathrm{K} \alpha 1-\mathrm{S} / \mathrm{K} \alpha 2-\mathrm{S}$ of $0.435 \pm 0.030$ corresponds nicely to the calculated $\mathrm{L}_{3 / 2} / \mathrm{L}_{1 / 2}-\mathrm{REC}$ cross section ratio of 0.420 . Using the theoretical total $\mathrm{L}_{\mathrm{j}}$-REC cross sections, the RTE contributions to the line intensities can be extracted directly. Both REC and RTE calculations agree quite nicely with the experimental findings.

\section{CONCLUSIONS}

For the heaviest and simplest atomic system resonant transfer and excitation involving the innermost shells have been investigated. We measured for $\mathrm{H}$-like $\mathrm{U}^{91+}$ projectiles the $\mathrm{KL}_{\mathrm{j}} \mathrm{L}_{\mathrm{j}}$ - $\mathrm{RTE}$ using a $\mathrm{H}_{2}$ gas target providing the narrowest possible Compton profile for atomically confined quasi-free target electrons. We studied the emission patterns of the cascade decay of doubly excited He-like $\mathrm{U}^{90+* *}$ ions where the first hypersatellite (Koi-

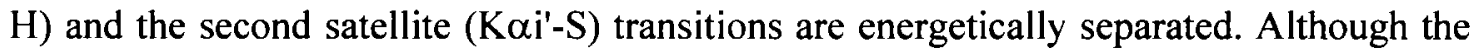

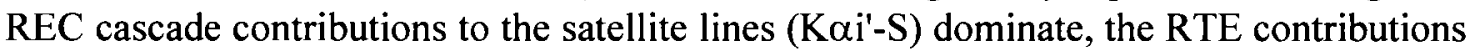
could be isolated. In particular, the hypersatellite $(\mathrm{K} \alpha \mathrm{i}-\mathrm{H})$ transitions are free of REC contributions - neglecting possible small interference effects.

The emission patterns for the satellite lines, for $\mathrm{K} \alpha 2-\mathrm{S}$ as well as for $\mathrm{K} \alpha \mathrm{l}-\mathrm{S}$, are found to be isotropic. However, these transitions are heavily determined by the REC cascade contributions, which also have been found to be isotropic. The latter is in clear contrast to the emission for REC cascades observed recently with bare $\mathrm{U}^{92+}$ projectiles [33]; this point will be considered elsewhere. The anisotropies predicted for the satellite contributions caused by RTE cascades cannot be observed for our case within the experimental uncertainties. However, the hypersatellite emission is free of REC cascade 
contributions: For the first resonance 1-R $\left(\mathrm{KL}_{1 / 2} \mathrm{~L}_{1 / 2}-\mathrm{RTE}\right)$ isotropic emission for the $\mathrm{K} \alpha 2-\mathrm{H}$ emission is found as predicted. For the second resonance group $2-\mathrm{R}\left(\mathrm{KL}_{1 / 2} \mathrm{~L}_{3 / 2}-\right.$ $\mathrm{RTE})$ the $\mathrm{K} \alpha \mathrm{l}-\mathrm{H}$ is strongly anisotropic in overall accordance with theory pointing to a complete alignment of the $\mathrm{j}=3 / 2$ electrons. However, the experiment shows a large alignment parameter of $\boldsymbol{\beta}_{A}=-0.75$ exceeding the predicted value. In how far this enhancement may be caused by higher multipole contributions has to be further investigated. At 2-R, for the $\mathrm{K} \alpha 2-\mathrm{H}$ emission isotropy is observed - possibly with a slight tendency to an opposite anisotropy - and this agrees with predictions. At the third resonance group 3-R $\left(\mathrm{KL}_{3 / 2} \mathrm{~L}_{3 / 2}-\mathrm{RTE}\right)$ no anisotropy could be found for the $\mathrm{K} \alpha 1-\mathrm{H}$ emission, also in accordance with theory.

The measured intensity ratios for the prominent $x$-ray lines fit also to the expectations and were used to normalize the RTE cascade contributions to the satellite lines. From the measured angular distributions total cross sections for hypersatellite emission caused by RTE were reported. Normalizing these relative cross sections using the K-REC emission gives absolute values, which are in agreement with the fully relativistic calculations including the full Breit interaction. For the first resonance group the Breit interaction increases the cross section by a factor of two. In summary a general good agreement between fully relativistic theory and experiment can be stated for pure electron-electron interaction in the strongest possible atomic fields. This is true for both the total as well as the angular differential x-ray emission.

\section{Acknowledgements}

The close and good collaboration with our colleagues from the theoretical physics department at the University of Giessen under the leadership of Werner Scheid is highly appreciated; especially we name additionally Stephan Zakowicz, Zoltán Harman and Norbert Grün. We like to thank also Jörg Eichler and his colleague Akira Ichihara for providing us with calculations for the REC contributions. We are indebted to the ESR team, Markus Steck and his colleagues, for providing us with an excellent beam, to Otto Klepper for his assistance with the particle detector, to Uli Popp for his help with the gas jet operation, and to the colleagues from the atomic physics group supporting the beam time. Supports and grants from EU (EC-HPRI-CT-1999-0001; HPMT-CT-2000-00179) and from WTZ, Bairen Plan and NSFC are highly acknowledged.

\section{REFERENCES:}

[1] D.R. Bates, Case Studies in Atomic Physics 4 (1974) 62

[2] B.L. Whitten, A.U. Hazi, M.H. Chen, P.L. Hagelstein. Phys.Rev. A33 (1986) 2171

[3] S. Baird, J. Bosser, C. Carli et al., Phys. Lett. B361 (1995) 184

[4] A. Burgess, Astrophys.J. 139 (1964) 776

[5] C. Brandau, T. Bartsch, K. Beckert et al., Nucl.Instr.Meth. B (2003) in print (HCI-2002) 
[6] P. Zimmerer, N. Grün, W. Scheid; Phys. Lett. A148 (1990) 457

[7] M. H. Chen, Phys. Rev. A41 (1990) 4102

[8] M. S. Pindzola, N. R. Badnell, Phys. Rev. A42 (1990) 6526

[9] G. Soff, T. Beier, M. Greiner et al., Adv.Quant.Chem. 30 (1998) 125

[10] C. Brandau, S. Böhm, C. Kozhuharov et al., GSI Scientific Report 2002 (GSI-Rep 2003-1) 91 \{http://www.gsi.de/annrep2002\}

[11] J.A. Tanis, W.G. Graham, K.H. Berkner et al., Nucl.Instr.Meth. B53 (1991) 442

[12] W.G. Graham, K.H. Berkner, E.M. Bernstein et al., Nucl.Instr.Meth.B53 (1990) 2773

[13] P.H. Mokler, S. Reusch, Z.Phys. D8 (1988) 393

[14] T. Kandler, P.H. Mokler, Th. Stöhlker et al., Phys.Lett. A204 (1995) 274

[15] T. Kandler, Th. Stöhlker, P.H. Mokler et al., Z.Phys. D35 (1995) 15

[16] T. Kandler, P.H. Mokler, H. Geissel et al., Nucl.Instr.Meth. B98 (1995) 320

[17] P.H. Mokler, T. Kandler, Th. Stöhlker et al., Physica Scripta T73 (1997) 247

[18] M. Gail. N. Grün, W. Scheid, J.Phys. B31 (1998) 4645

[19] M.H. Chen, J.H. Scofield, Phys.Rev. A52 (1995) 2057

[20] X. Ma, P.H. Mokler, G. Bednarz et al., Nucl.Instr.Meth. B (2003) in print (HCI2002)

[21] Th. Stöhlker, X. Ma, T. Ludziejewski et al., Phys.Rev.Lett. 86 (2001) 983

[22] S. Zakowicz, Z. Harman, N. Grün, W. Scheid, Phys.Rev. A (joint to this issue)

[23] K.G. Dyall, I.P. Grant, C.T. Johnson et al., Comput.Phys.Commun. 55 (1989) 425

[24] M. Gail, PhD thesis - University Giessen and private communication (2000)

[25] A. Krämer, A. Kritzer, H. Reich, Th. Stöhlker, Nucl.Instr.Meth. B174 (2001) 205

[26] O. Klepper, Nucl.Phys. A626 (1997) 199c

[27] Th. Stöhlker, T. Ludziejewski, F. Bosch et al., Phys.Rev.Lett. 82 (1999) 3232

[28] Th. Stöhlker, P.H. Mokler, C. Kozhuharov, A. Warczak, Comments At.Mol.Phys. 33 (1997) 271

[29] G. Bednarz, A. Warczak, D. Sierpowski et al., Nucl.Instr.Meth. B (2003) in print (APAC)

[30] Th. Stöhlker, F. Bosch, A. Gallus et al., Phys.Rev.Lett. 79 (1997) 3270

[31] A. Ichihara, T. Shirai, J. Eichler K-REC, Phys.Rev. A54 (1996) 4954

[32] J. Eichler, A. Ichihara, private communication (2002)

[33] A. Surzhykov, S. Fritzsche, A. Gumberidze, Th. Stöhlker, Phys.Rev.Lett. 88 (2002) 153001

[34] M. Gail, N. Grün, W. Scheid, J.Phys. B31 (1998) 4645 
Table 1: Experimental parameters for $\mathrm{x}$-ray detection

\begin{tabular}{cccccccc}
\hline $\begin{array}{c}\text { Lab } \\
\text { angles } \\
(\mathrm{deg})\end{array}$ & $\begin{array}{c}\text { detector } \\
\text { size } \\
(\mathrm{mm})\end{array}$ & $\begin{array}{c}\text { slit } \\
\text { dim. } \\
(\mathrm{mm})\end{array}$ & $\begin{array}{c}\text { solid } \\
\text { angle } \\
\left(10^{-4} \mathrm{sr}\right)\end{array}$ & $\begin{array}{c}\text { window } \\
(\mathrm{A}-\mu \mathrm{m})\end{array}$ & $\begin{array}{c}\text { efficiency } \\
\text { @100keV ion frame } \\
{[\mathrm{lab} \text { energy, keV] }}\end{array}$ & $\begin{array}{c}\text { resolution (eV }) \\
{[@ \text { x-ray energy, }} \\
\text { keV] }\end{array}$ \\
\hline 13.3 & $30 \times 13$ & -- & 14.1 & $\mathrm{Fe}-50$ & 0.38 & {$[@ 163.0]$} & $720[@ 160.6]$ \\
14.7 & $30 \times 13$ & -- & 13.9 & $\mathrm{Fe}-50$ & 0.38 & {$[@ 162.2]$} & $740[@ 160.6]$ \\
35 & $\Phi 44$ & $4 \times 44$ & 13.6 & $\mathrm{Fe}-50$ & 0.71 & {$[@ 143.7]$} & $900[@ 59.5]$ \\
60 & $\Phi 50$ & $3 \times 50$ & 8.79 & $\mathrm{Be}-100$ & 0.63 & {$[@ 115.4]$} & $640[@ 59.5]$ \\
90 & $\Phi 25$ & $4 \times 25$ & 4.19 & $\mathrm{Be}-100$ & 0.75 & {$[@ 88.2]$} & $600[@ 59.5]$ \\
120 & $\Phi 50$ & $3 \times 30$ & 3.39 & $\mathrm{Be}-100$ & 0.87 & {$[@ 71.3]$} & $830[@ 59.5]$ \\
150 & $\Phi 25$ & $10 \times 25$ & 16.9 & $\mathrm{Be}-100$ & 0.88 & {$[@ 62.6]$} & $490[@ 59.5]$ \\
\hline
\end{tabular}


Table 2: $\quad$ Relative line intensities (for details see text)

\begin{tabular}{|c|c|c|c|c|c|c|c|c|}
\hline$\Theta_{\mathrm{lab}}$ & K-REC & $\begin{array}{l}\mathrm{L}_{3 / 2-} \\
\mathrm{REC}\end{array}$ & $\begin{array}{l}\mathrm{L}_{1 / 2^{-}} \\
\mathrm{REC}\end{array}$ & $\mathrm{Ka} 2-\mathrm{S}$ & $\mathrm{Ka} 1-\mathrm{S}$ & $\mathrm{Ka} 2-\mathrm{H}$ & $\mathrm{Kal}-\mathrm{H}$ & $\begin{array}{l}\text { K-REC theory } \\
\text { (barn/sterad) }\end{array}$ \\
\hline \multicolumn{9}{|c|}{ Off-resonance at $102.0 \mathrm{MeV} / \mathrm{u}$} \\
\hline 13.3 & 1876 & 729 & 1808 & 11641 & 5369 & -- & -- & 3.02 \\
\hline 14.7 & 1934 & 862 & 2102 & 11347 & 5012 & -- & -- & 3.21 \\
\hline 35 & 4972 & 432 & 3757 & 9819 & 4195 & -- & -- & 7.41 \\
\hline 60 & 6753 & 965 & 3148 & 4455 & 1948 & -- & -- & 12.69 \\
\hline 90 & 3123 & 796 & 1517 & 1375 & 573 & -- & -- & 13.83 \\
\hline 120 & 2155 & -- & -- & 839 & 321 & -- & -- & 9.04 \\
\hline 150 & 2600 & 944 & 1459 & 2289 & 1088 & -- & -- & 2.79 \\
\hline \multicolumn{9}{|c|}{ Resonance group 1 at $116.6 \mathrm{MeV} / \mathrm{u}$} \\
\hline 13.3 & 1335 & 2569 & 1578 & 9964 & 3490 & 2618 & -- & 2.64 \\
\hline 14.7 & 1266 & 2353 & 1335 & 9742 & 3355 & 2314 & -- & 2.80 \\
\hline 35 & 3449 & 1803 & 1803 & 8983 & 2771 & 1803 & -- & 6.31 \\
\hline 60 & 4707 & 1102 & 1962 & 3866 & 1265 & 1102 & -- & 10.77 \\
\hline 90 & 2312 & 251 & 251 & 1037 & 368 & 251 & -- & 11.72 \\
\hline 120 & 1598 & -- & -- & -- & - & -- & -- & 7.65 \\
\hline 150 & 1683 & 445 & 445 & 1946 & 685 & 445 & -- & 2.35 \\
\hline \multicolumn{9}{|c|}{ Resonance group 2 at $124.9 \mathrm{MeV} / \mathrm{u}$} \\
\hline 13.3 & 4355 & 940 & 2332 & 30002 & 13506 & 2246 & 2059 & 2.46 \\
\hline 14.7 & 4897 & 1037 & 2529 & 29197 & 13471 & 2092 & 2249 & 2.61 \\
\hline 35 & 12042 & -- & -- & -- & -- & -- & -- & 5.81 \\
\hline 60 & 17304 & 2282 & 7446 & 10529 & 4771 & 535 & 1579 & 9.89 \\
\hline 90 & 7813 & 1465 & 2791 & 3085 & 1485 & 133 & 438 & 10.76 \\
\hline 120 & 5600 & -- & -- & -- & -- & -- & -- & 7.02 \\
\hline 150 & 5537 & 2171 & 3356 & 5161 & 2509 & 413 & 513 & 2.16 \\
\hline \multicolumn{9}{|c|}{ Resonance group 3 at $133.1 \mathrm{MeV} / \mathrm{u}$} \\
\hline 13.3 & 1032 & 265 & 657 & 7048 & 3224 & -- & 311 & 2.31 \\
\hline 14.7 & 1006 & 262 & 640 & 6859 & 3076 & -- & 272 & 2.44 \\
\hline 35 & 2994 & -- & -- & -- & -- & -- & -- & 5.37 \\
\hline 60 & 4463 & 532 & 1735 & 2420 & 1087 & -- & 72 & 9.13 \\
\hline 90 & 2112 & -- & -- & -- & -- & -- & -- & 9.93 \\
\hline 120 & 1287 & -- & -- & -- & -- & -- & -- & 6.47 \\
\hline 150 & 1339 & 556 & 859 & 1086 & 558 & -- & 44 & 2.00 \\
\hline
\end{tabular}


Table 3: Total theoretical x-ray emission cross sections in barn (per quasi-free target electron) induced by RTE according to Ref. [22].

\begin{tabular}{lrrr}
\hline b] & \multicolumn{1}{c}{ 1-R } & $2-\mathrm{R}$ & $3-\mathrm{R}$ \\
\hline $\mathrm{K} \alpha 2-\mathrm{S}$ & 21.72 & 8.81 & 0.47 \\
$\mathrm{~K} \alpha 2-\mathrm{H}$ & 21.70 & 4.22 & 0.06 \\
$\mathrm{~K} \alpha 1-\mathrm{S}$ & 0.01 & 3.35 & 2.48 \\
$\mathrm{~K} \alpha 1-\mathrm{H}$ & 0.03 & 7.94 & 2.90 \\
\hline
\end{tabular}

Table 4: Total hypersatellite emission cross sections (per target electron) in comparison to the corresponding RTE cross sections. The theoretical values are from Ref. [22]; the numbers in brackets give the results of calculations without Breit interaction.

\begin{tabular}{lcccc}
\hline & $1-\mathrm{R}$ & \multicolumn{2}{c}{$2-\mathrm{R}$} & $3-\mathrm{R}$ \\
& $\mathrm{K} \alpha 2-\mathrm{H}$ & $\mathrm{K} \alpha 2-\mathrm{H}$ & $\mathrm{K} \alpha 1-\mathrm{H}$ & $\mathrm{K} \alpha 1-\mathrm{H}$ \\
\hline experiment & $22.4 \pm 2.7$ & $4.5 \pm 1.5$ & $7.2 \pm 0.8$ & $2.5 \pm 0.8$ \\
theory & 21.7 & 4.2 & 7.9 & 2.9 \\
& $(12.0)$ & $(4.5)$ & $(7.2)$ & $(2.4)$ \\
\hline
\end{tabular}




$\begin{array}{cll}\mathrm{RTE} & \mathrm{REC} \text { and } \\ \mathrm{KL}_{1 / 2} \mathrm{~L}_{1 / 2} & \text { Stabilization } & \text { Cascades }\end{array}$

(a)

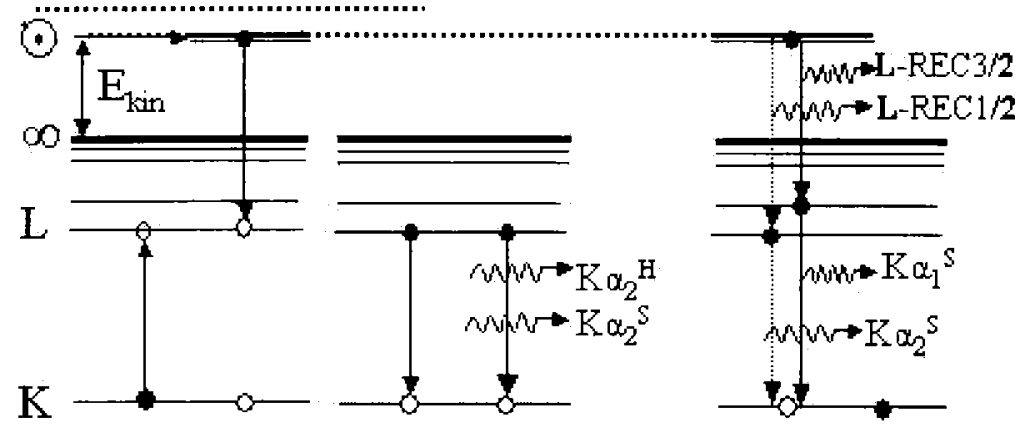

$(1 / 2,1 / 2)$

1-R

K

$\mathrm{KL}_{1 / 2} \mathrm{~L}_{3 / 2}$

(b)

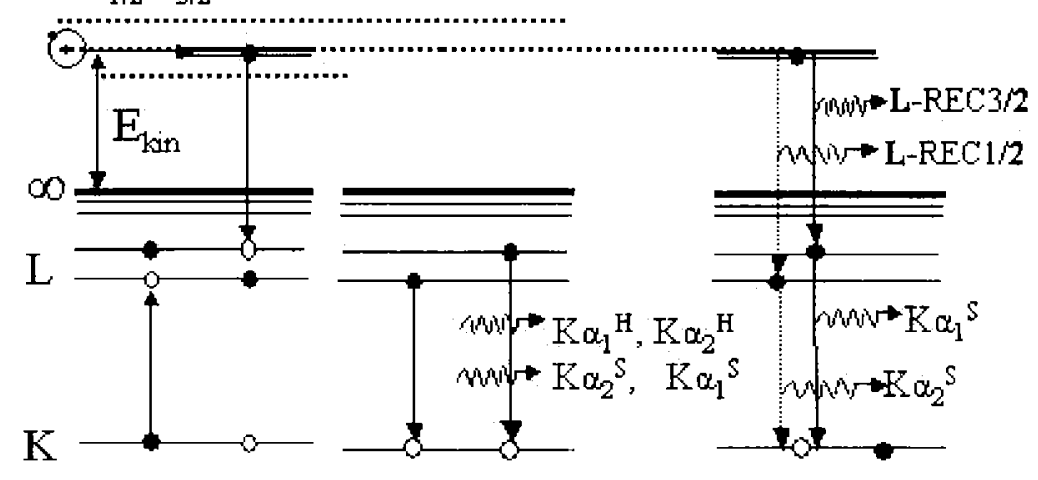

$(1 / 2,3 / 2)$

2-R

$(1 / 2,3 / 2)$

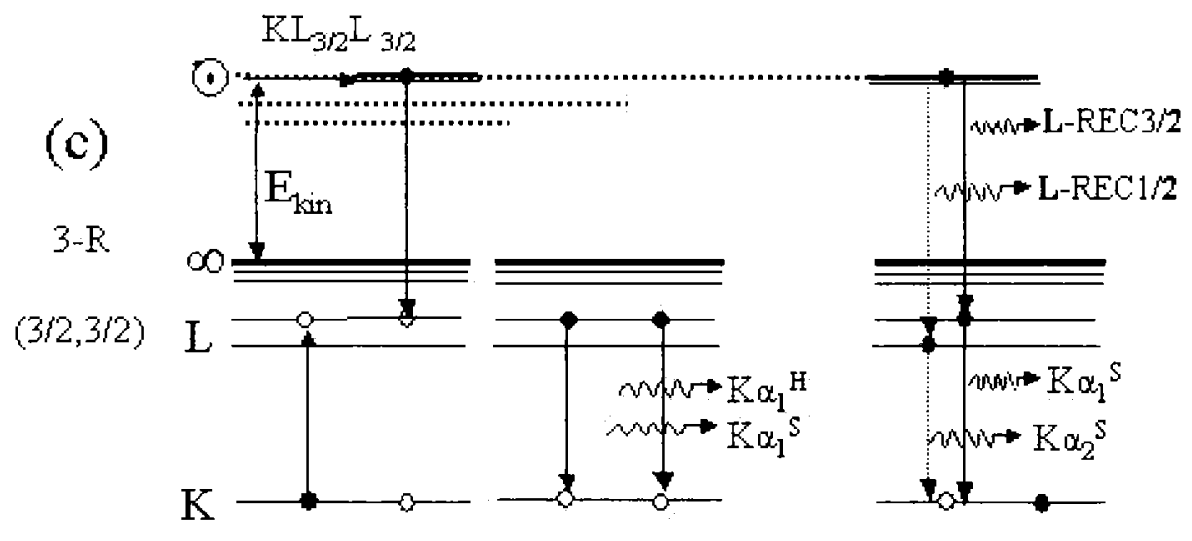

Fig. 1: Explanation of the $\mathrm{KL}_{\mathrm{j}} \mathrm{L}_{\mathrm{j}}$ - $\mathrm{RTE}$ process; the level diagrams on the right hand side give the competing $\mathrm{L}_{\mathrm{j}}$ - REC process for comparison. 


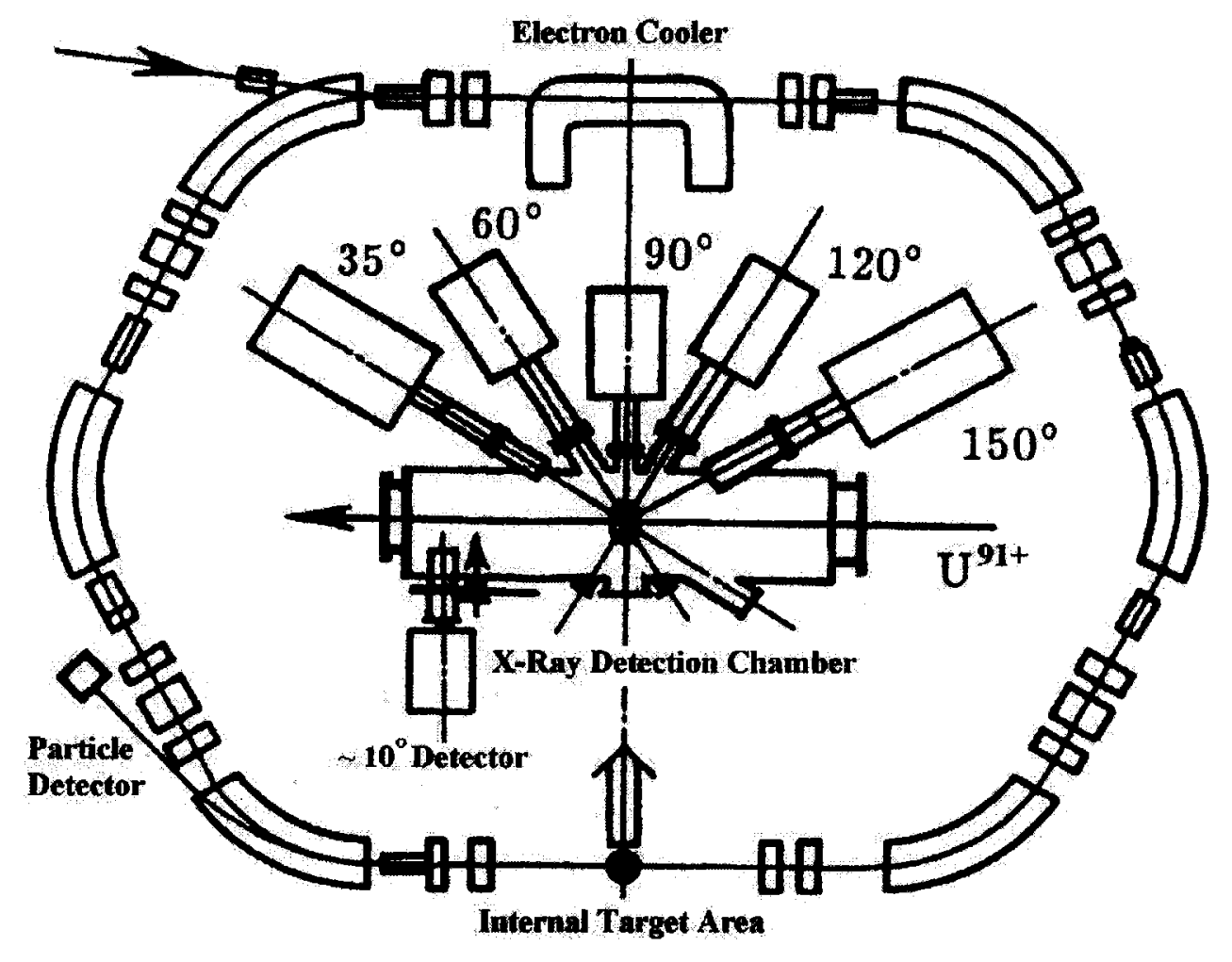

Fig. 2: Experimental arrangement at the heavy ion storage-cooler ring ESR. The insert in the center gives details of the $\mathrm{x}$-ray detection chamber around the gas-jet target area. 


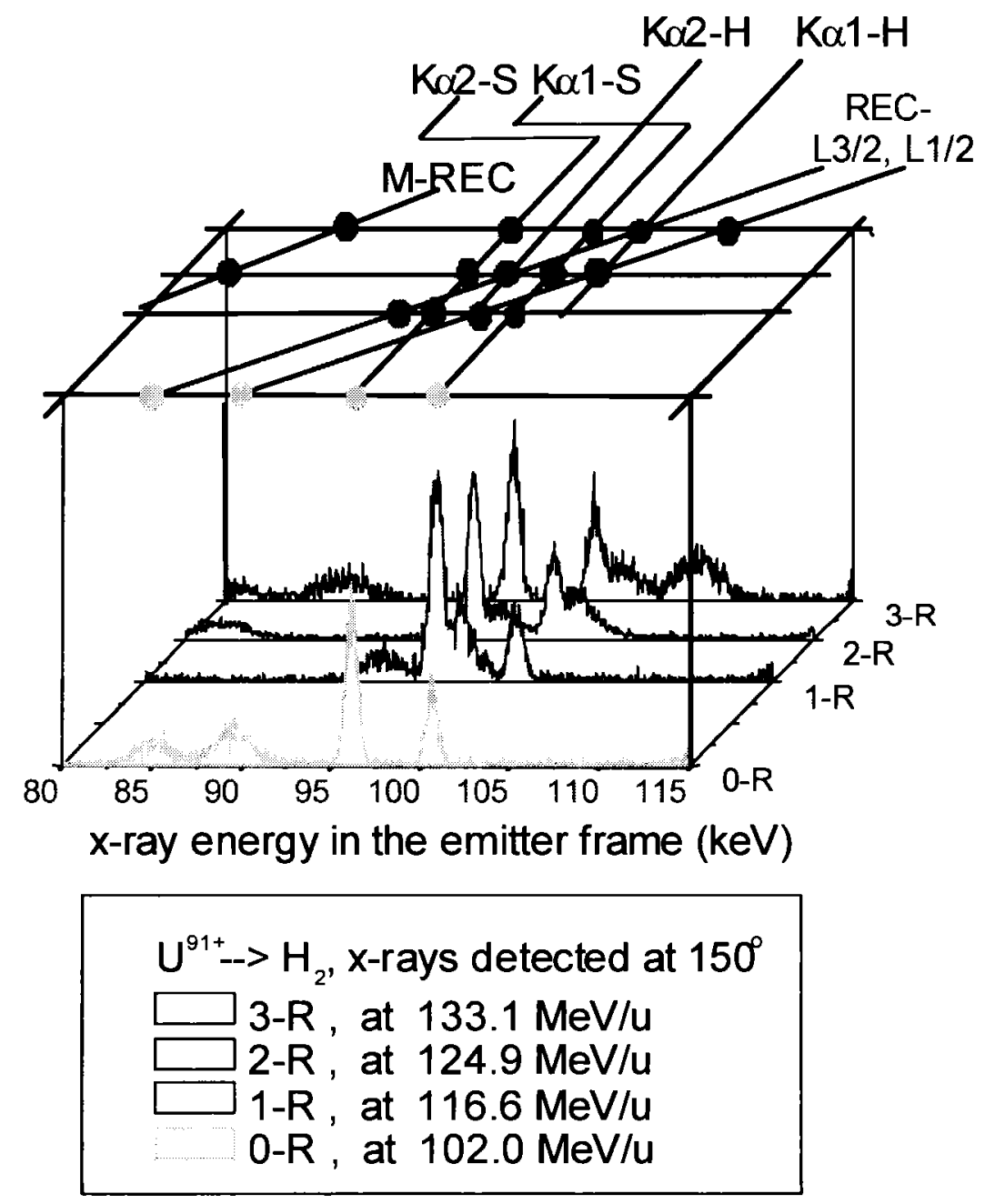

Fig. 3: X-ray spectra associated with one electron capture for $\mathrm{U}^{91+}$ on $\mathrm{H}_{(2)}$ at 102.0 $\mathrm{MeV} / \mathrm{u}(\mathrm{O}-\mathrm{R}), 126.6 \mathrm{MeV} / \mathrm{u}(1-\mathrm{R}), 124.9 \mathrm{MeV} / \mathrm{u}(2-\mathrm{R})$, and 133.1 MeV/u (3-R) taken at an observation angle of $150^{\circ}$. In the plane at the top of the figure the different transitions are indicated. 


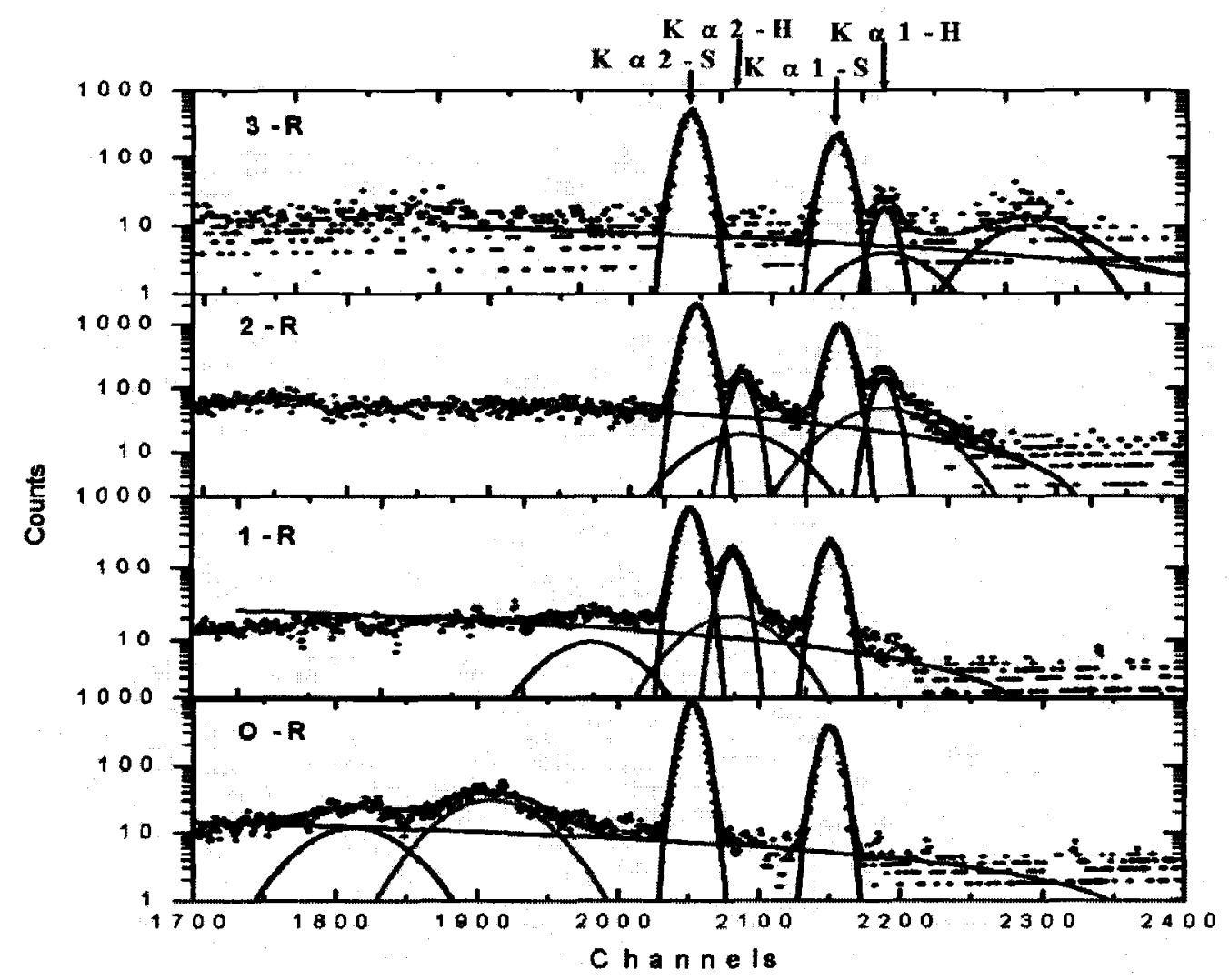

Fig. 4: Decomposition of the $\mathrm{L}$ x-ray spectra into the various components. The channels have been shifted in such a way that characteristic lines are aligned visually 


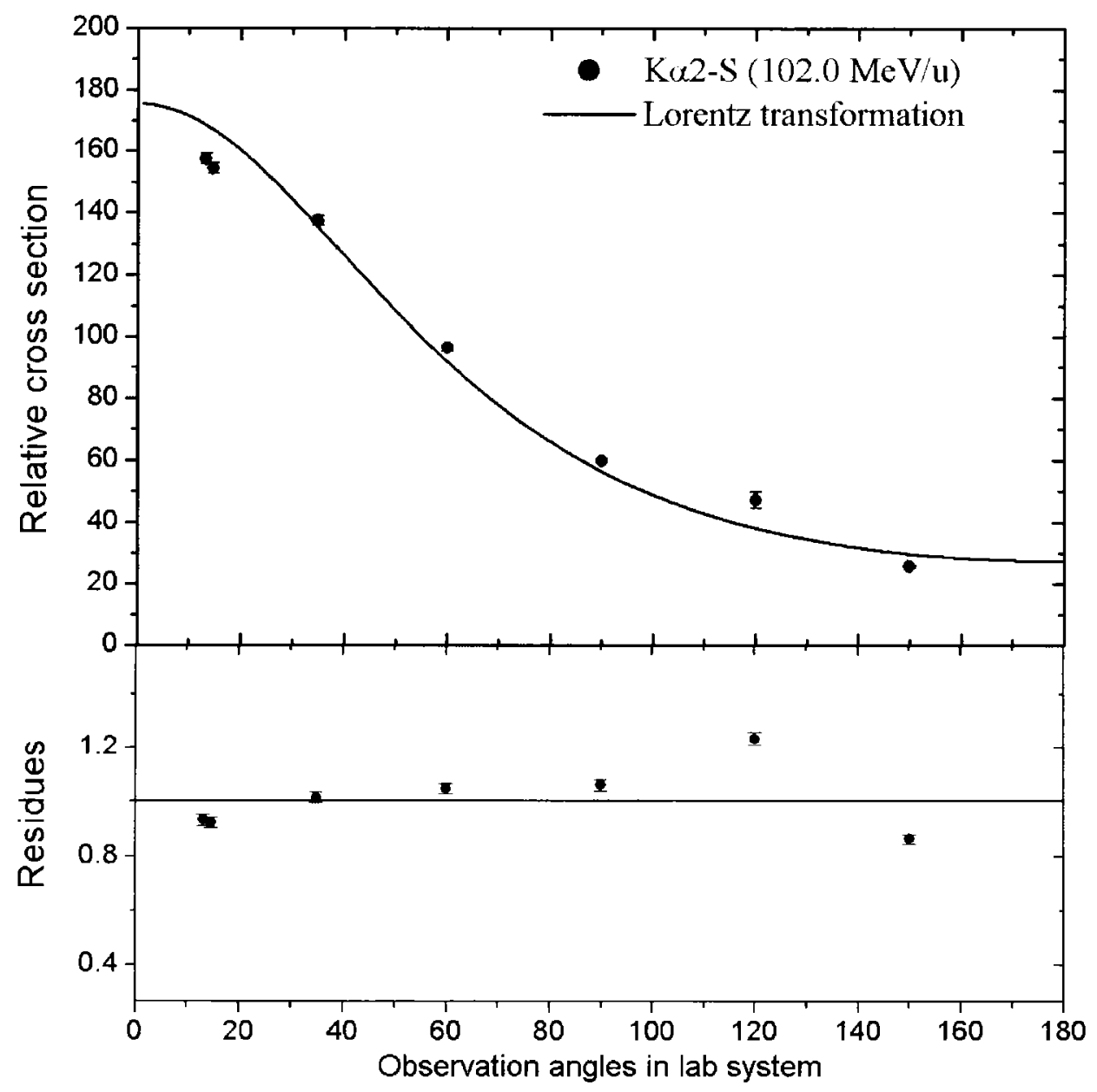

Fig. 5: The emission characteristic of the Ka2-S line as observed in the laboratory at the $\mathrm{O}-\mathrm{R}$ energy of $102.0 \mathrm{MeV} / \mathrm{u}$. The solid line gives the Lorentz transformation for an isotropic emission in the emitter frame. At the bottom the ratio $\mathrm{K} \alpha 2-\mathrm{S} /$ Lorentz transformation is given. 


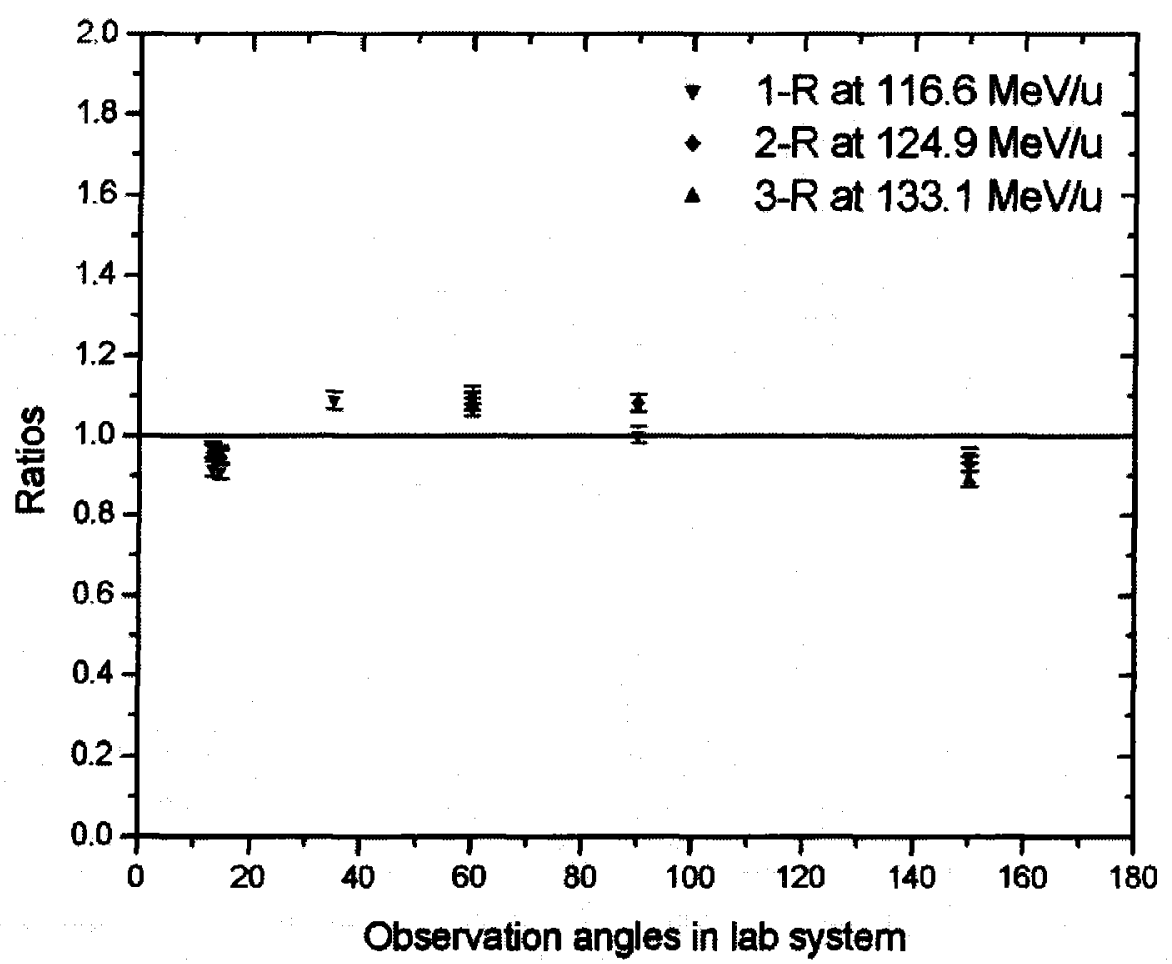

Fig. 6: The ratios for the $\mathrm{K} \alpha 2-\mathrm{S}$ emission to the expectation of an isotropic emission in the emitter frame for the three resonance groups 1-R (116.6 MeV/u), 2-R (124.9 MeV/u), and 3-R (133.1 MeV/u). 


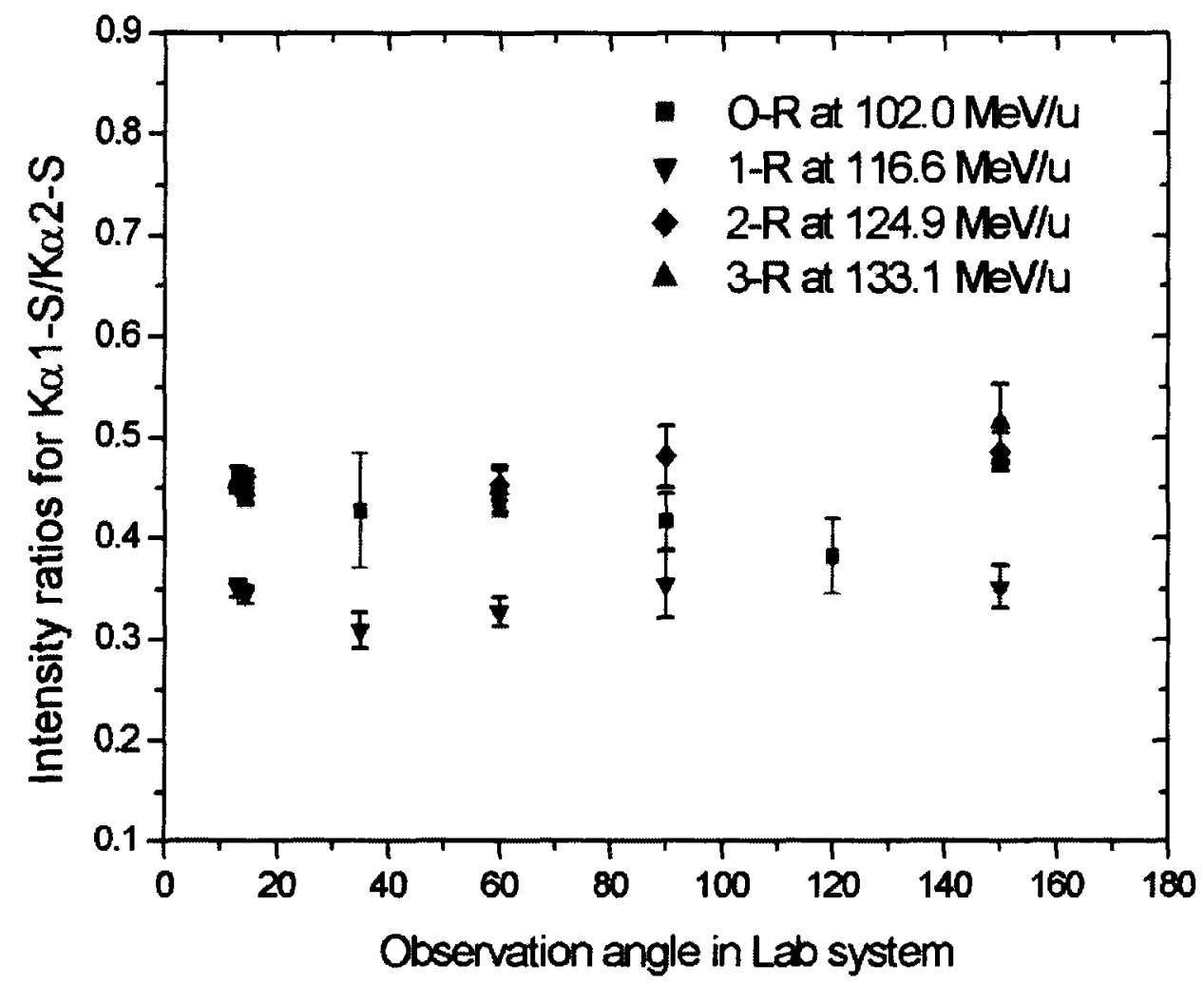

Fig. 7: K $\alpha 1-\mathrm{S} / \mathrm{K} \alpha 2-\mathrm{S}$ intensity ratios as a function of the emission angle for all the measured cases $\mathrm{O}-\mathrm{R}, \mathrm{1}-\mathrm{R}, 2-\mathrm{R}$, and 3-R. 


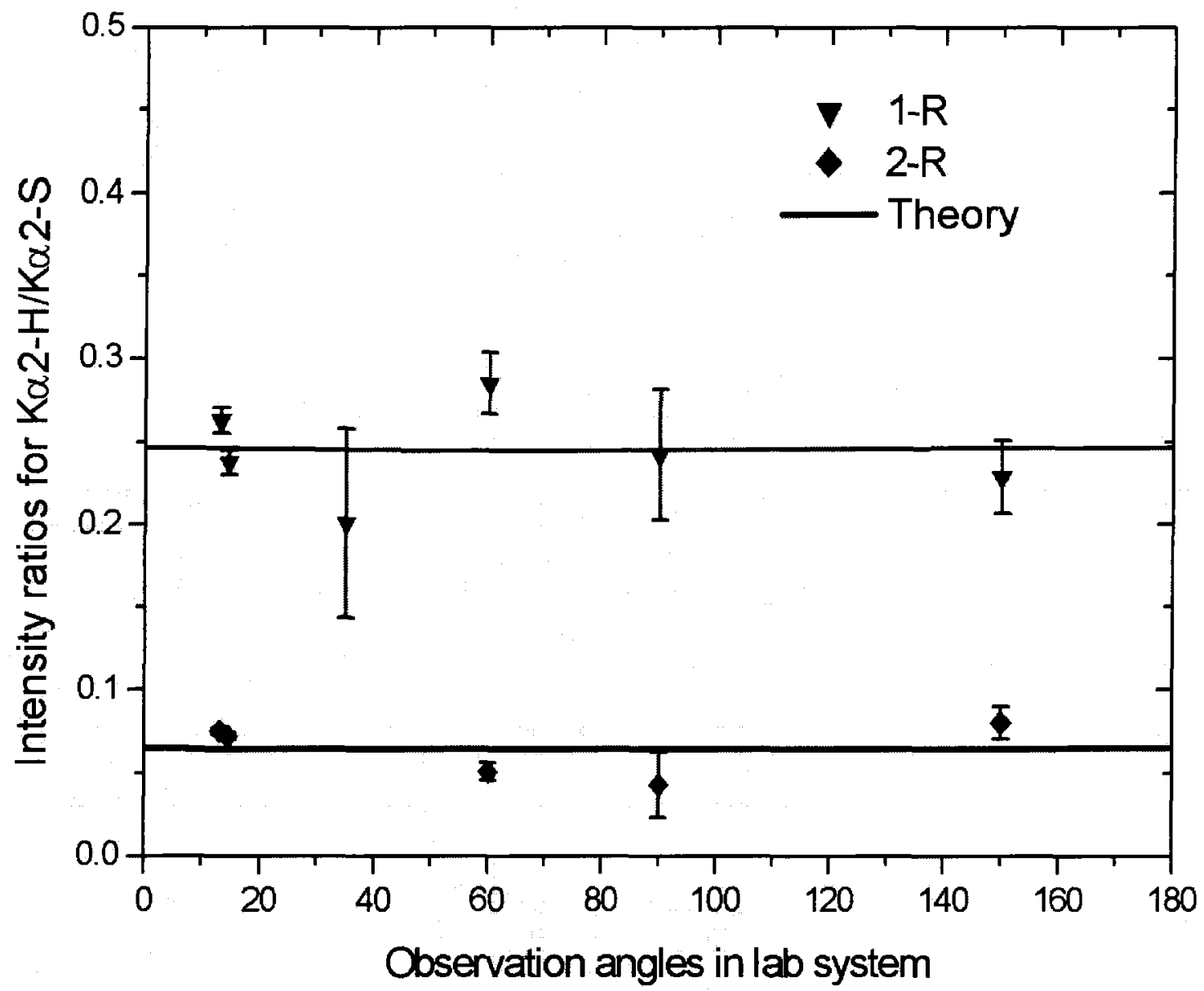

Fig. 8: K $\alpha 2-\mathrm{H} / \mathrm{K} \alpha 2-\mathrm{S}$ intensity ratios for the first and second RTE resonance groups $1-\mathrm{R}$ and 2-R. The full lines give the theoretical predictions according to Ref. [22]. 


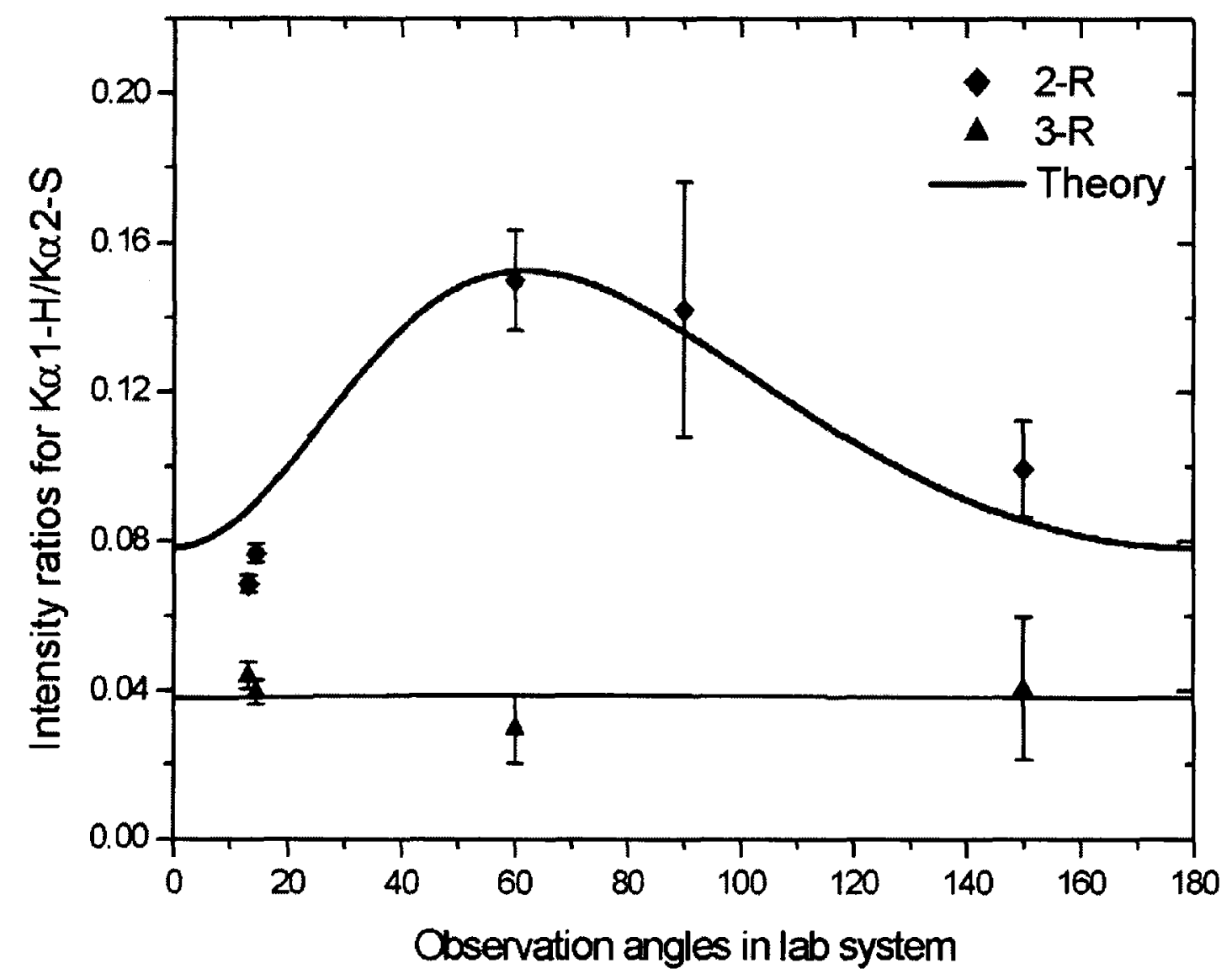

Fig. 9: $\mathrm{K} \alpha 1-\mathrm{H} / \mathrm{K} \alpha 2-\mathrm{S}$ intensity ratios for the second and third resonance groups 2-R and 3-R. The full lines are the theoretical results according to Ref. [22]. 


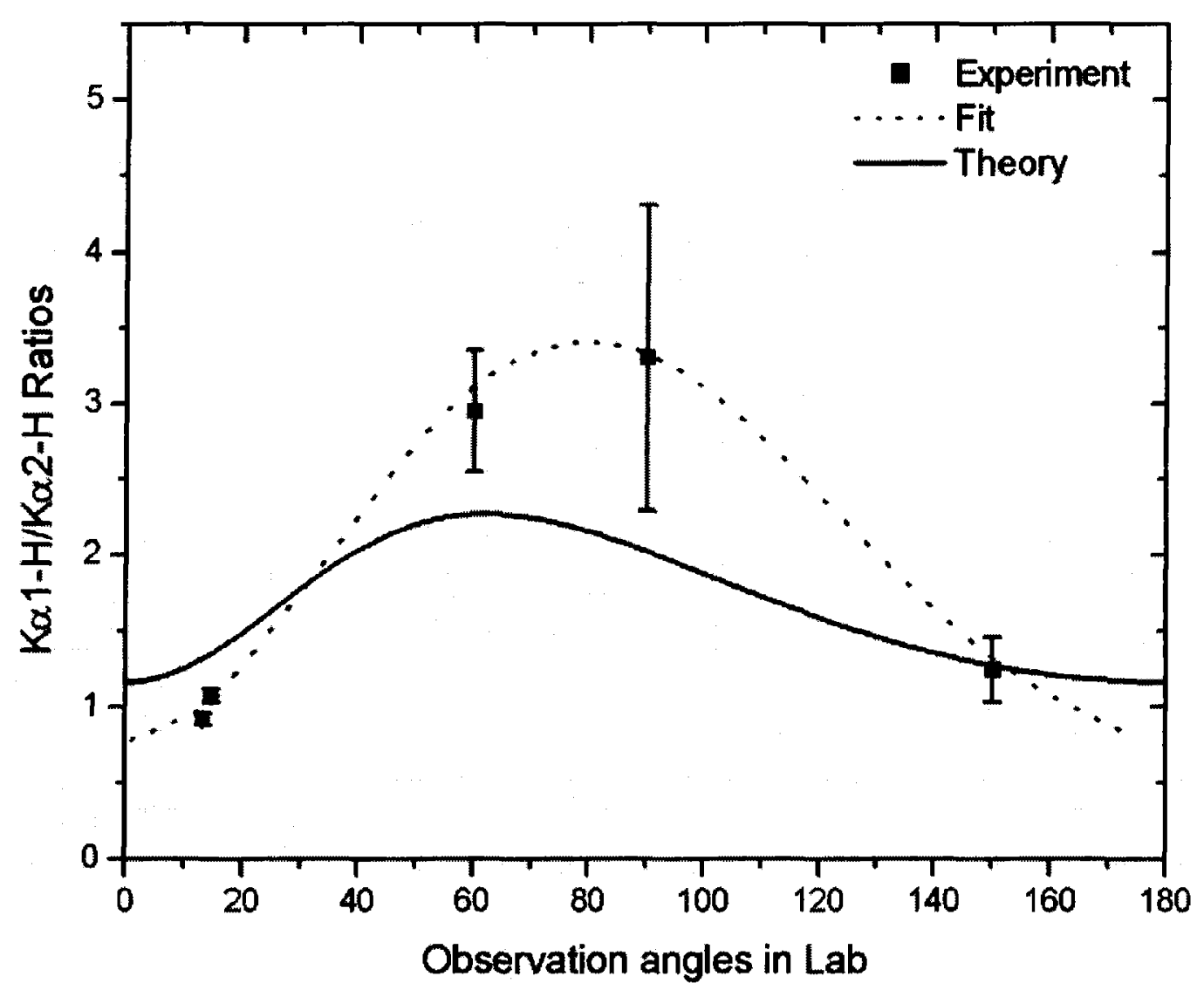

Fig. 10: K $\alpha 1-\mathrm{H} / \mathrm{K} \alpha 2-\mathrm{H}$ intensity ratios for the second resonance group 2-R. The full lines give the theoretical results according to [22]; the dotted line is a fit to the experimental data. 



\title{
Angular distribution of hypersatellite and satellite radiation emitted after resonant transfer and excitation into $\mathrm{U}^{91+}$ ions
}

\author{
S. Zakowicz, Z. Harman, ${ }^{*}$ N. Grün, and W. Scheid ${ }^{\dagger}$ \\ Institut für Theoretische Physik der Justus-Liebig-Universität Giessen, \\ Heinrich-Buff-Ring 16, D-35392 Giessen, Germany
}

(Dated: August 6, 2003)

\begin{abstract}
In collisions of heavy few-electron projectile ions with light targets, an electron can be transferred from the target with the simultaneous excitation of a projectile electron. We study the angular distribution of de-excitation $\mathrm{X}$ rays following the resonant capture process. Our results are compared to experimental values of Ma et al. [Phys. Rev. A (joint to this issue)] for collisions of $\mathrm{U}^{91+}$ ions with a hydrogen gas target.

PACS numbers: $34.70 .+\mathrm{e}, 32.80 . \mathrm{Hd}, 31.30 . \mathrm{Jv}, 34.80 . \mathrm{Lx}$
\end{abstract}

Keywords: Resonant transfer and excitation, X-ray angular distribution, impulse approximation

\footnotetext{
*Electronic address: zoltan.harman@theo.physik.uni-giessen.de

${ }^{\dagger}$ http://service.physik.uni-giessen.de
} 


\section{INTRODUCTION}

Electron dynamics and interactions are strongly influenced in very heavy atomic systems by relativistic effects. The investigation of resonant transfer and excitation (RTE) in highlycharged ions has proved to be a suitable tool to study these phenomena. In this process, a quasi-free electron from a low- $Z$ target atom is captured into an ion with the simultaneous excitation of a projectile electron, followed by the emission of stabilizing characteristic X-ray photons. The RTE process is closely related to dielectronic recombination (DR), in which an initially free electron is captured.

Relativistic effects are most pronounced for $K L L$ transitions, where inner-shell electrons are involved. For hydrogen-like ions, the whole process including de-excitation by photon emission may be written as

$$
1 s_{1 / 2}+e^{-} \rightarrow\left[2 l_{j} 2 l_{j^{\prime}}^{\prime}\right]_{d_{1}} \rightarrow\left[1 s_{1 / 2} 2 l_{j^{\prime \prime}}^{\prime \prime}\right]_{d_{2}}+K \alpha^{\mathrm{HS}} \rightarrow 1 s^{2}+K \alpha^{\mathrm{HS}}+K \alpha^{\mathrm{S}}
$$

Here, the first intermediate state $d_{1}$ decays into $d_{2}$ by emission of a hypersatellite (HS) photon, and then the ground state of the helium-like system is reached by a satellite (S) transition. (For simplicity we omitted most reaction pathways with emission of more than two photons.) Alternatively, the second intermediate states $d_{2}$ can also be reached by radiative electron capture (REC), that is, capture with emission of a photon. $\mathrm{X}$ rays emitted following REC into the $L$-subshell levels give a sizeable contribution to the measured satellite intensities.

A similar RTE process was studied both experimentally [2] and theoretically [3] in the case of initially helium-like uranium ions, where only one $K \alpha$ photon is emitted. The emission has been shown to be anisotropic due to the non-uniform occupation of the magnetic sublevels (the so-called alignment) of the states formed by resonant capture. It was also shown in [3] that the Breit term of the electron-electron interaction gives an observable important contribution to the parameters characterizing the anisotropy of dipole emission. Balashov et al. [4] calculated general expressions for the angular anisotropy and correlation of cascade photons emitted in the DR and RTE processes. These authors supply numerical results only for a low-Z system, and their computations have not been experimentally verified yet.

In this paper we present calculations for the angular distributions of HS and S photons emitted during collisions of $\mathrm{U}^{91+}$ ions with a hydrogen gas target. The resulting intensity 
ratios are compared to those measured by Ma et al. [1]. Due to the narrow Compton profile of this target, the resonances in the cross sections have relatively small widths and can be resolved experimentally. In particular, the angular distribution of the radiation is only slightly blurred by overlapping resonances.

Atomic units will be used throughout.

\section{THEORY}

\section{A. Two-photon emission in the DR process}

In reference [5], the triple-differential cross section for DR with two photon emissions is found to be

$$
\frac{d^{3} \sigma_{\mathrm{DR}}}{d \omega_{1} d \Omega_{1} d \Omega_{2}}\left(\omega_{1} ; \theta_{1}, \phi_{1}, \theta_{2}, \phi_{2}\right)=\sum_{\substack{j, k=1 \\ j \neq k}}^{2} \sum_{d_{1}, d_{2}} \frac{d \sigma_{\mathrm{DR}}^{j, k}}{d \omega_{1}}\left(\omega_{1} ; d_{1}, d_{2}\right) W_{d_{1}, d_{2}}^{j, k}\left(\theta_{1}, \phi_{1}, \theta_{2}, \phi_{2}\right)
$$

The angles $\left(\theta_{1}, \phi_{1}\right) \equiv \Omega_{1}$ and $\left(\theta_{2}, \phi_{2}\right) \equiv \Omega_{2}$ give the directions into which the photons are emitted. The $z$ axis is chosen in the direction of the electron beam. The expression (2) is to be understood in such a way that the total DR cross section is

$$
\sigma_{\mathrm{DR}}=\frac{1}{2} \int d \omega_{1} d \Omega_{1} d \Omega_{2} \frac{d^{3} \sigma_{\mathrm{DR}}}{d \omega_{1} d \Omega_{1} d \Omega_{2}}\left(\omega_{1} ; \theta_{1}, \phi_{1}, \theta_{2}, \phi_{2}\right)
$$

The summations in (2) are extended over the possible doubly-excited $\left(d_{1}\right)$ and singly-excited $\left(d_{2}\right)$ states as well as over the two possible time orders of photon emission. Note that the cross section (2) is differential with respect to only one of the photon energies, which is labeled here as $\omega_{1}$. The energy $\omega_{2}$ of the other photon is fixed by conservation of energy:

$$
\omega_{2}=E-E_{f}-\omega_{1}
$$

where $E$ is the initial energy of the total system, and $E_{f}$ denotes the energy of the final atomic state without photons. We stress also that, since the two photons are indistinguishable particles, the cross section (2) is symmetric with respect to an exchange of both the energies and angles of the photons:

$$
\frac{d^{3} \sigma_{\mathrm{DR}}}{d \omega_{1} d \Omega_{1} d \Omega_{2}}\left(\omega_{1} ; \theta_{1}, \phi_{1}, \theta_{2}, \phi_{2}\right)=\frac{d^{3} \sigma_{\mathrm{DR}}}{d \omega_{1} d \Omega_{1} d \Omega_{2}}\left(E-E_{f}-\omega_{1} ; \theta_{2}, \phi_{2}, \theta_{1}, \phi_{1}\right) .
$$

Indeed, the variable $\omega_{1}$ denotes only the energy of one of the two photons, which is not necessarily the one that is emitted first. 
The partial cross sections in (2) have the form

$$
\begin{aligned}
\frac{d \sigma_{\mathrm{DR}}^{j, k}}{d \omega_{1}}\left(\omega_{1} ; d_{1}, d_{2}\right)= & \frac{2 \pi^{2}}{p^{2}} \frac{A_{r}\left(d_{2}, f\right)}{\Gamma_{d_{2}}} \frac{A_{r}\left(d_{1}, d_{2}\right)}{\Gamma_{d_{1}}} V_{a}\left(d_{1}\right) \\
& \times \frac{\Gamma_{d_{1}} /(2 \pi)}{\left(E-E_{d_{1}}\right)^{2}+\Gamma_{d_{1}}^{2} / 4} \frac{\Gamma_{d_{2}} /(2 \pi)}{\left(E-E_{d_{2}}-\omega_{j}\right)^{2}+\Gamma_{d_{2}}^{2} / 4},
\end{aligned}
$$

where $\omega_{2}$ is determined according to (4). Here, $p$ is the momentum of the incoming electron, $E_{d_{1}}$ and $E_{d_{2}}$ are the energies of the discrete intermediate states, and $\Gamma_{d_{1}}$ and $\Gamma_{d_{2}}$ their total widths. The quantities $A_{r}$ are radiative transition rates between the bound atomic states, and $V_{a}\left(d_{1}\right)$ is the capture rate from the initial states $i$ into the state $d_{1}$ given by

$$
V_{a}\left(d_{1}\right)=\frac{2 \pi}{2\left(2 J_{i}+1\right)} \sum_{M_{i} m_{s}} \sum_{M_{d_{1}}} \int d \Omega_{p}\left|\left\langle d_{1} J_{d_{1}} M_{d_{1}}\left|V^{C}+V^{B}\right| i J_{i} M_{i}, \mathbf{p} m_{s}\right\rangle\right|^{2} \rho_{i}
$$

where $J_{i}$ and $J_{d_{1}}$ are the total angular momenta of the corresponding states and $\rho_{i}$ is the state density at the initial state. The interaction of electrons 1 and 2 by exchanging a virtual photon of frequency $\omega$ is described by the sum of the Coulomb and generalized Breit operators

$$
\begin{aligned}
V_{12}^{C} & =\frac{1}{\left|\mathbf{r}_{1}-\mathbf{r}_{2}\right|} \equiv \frac{1}{r_{12}}, \\
V_{12}^{B} & =-\alpha_{1} \alpha_{2} \frac{\cos \left(\omega r_{12}\right)}{r_{12}}+\left(\alpha_{1} \nabla_{1}\right)\left(\alpha_{2} \nabla_{2}\right) \frac{\cos \left(\omega r_{12}\right)-1}{\omega^{2} r_{12}} .
\end{aligned}
$$

The latter accounts for retardation effects and magnetic interaction of the two Dirac currents. As (6) shows, the order of magnitude of the energy-differential cross section is determined by branching ratios and capture rates, whereas the shape is given by the product of two Lorentz profiles. The expression (6) has been obtained by employing a projection operator formalism, which is given in [5] and will be presented in a future publication.

The functions $W_{d_{1}, d_{2}}^{j, k}$ in (2) give the angular dependence of the radiation of the cascades. They can be expanded in tensor products of spherical harmonics:

$$
W_{d_{1}, d_{2}}^{j, k}\left(\theta_{1}, \phi_{1}, \theta_{2}, \phi_{2}\right)=\frac{1}{4 \pi} \sum_{\nu} \sum_{\nu_{1}, \nu_{2}} \beta_{\left(\nu_{1}, \nu_{2}\right) \nu}^{d_{1}, d_{2}, j, k}\left\{\mathbf{Y}_{\nu_{1}}\left(\theta_{1}, \phi_{1}\right) \otimes \mathbf{Y}_{\nu_{2}}\left(\theta_{2}, \phi_{2}\right)\right\}_{\nu, 0}
$$

The coefficients $\beta$ depend on the capture and radiative matrix elements, the partial wave phases of the incoming electron, and the involved angular momenta. The explicit analytical form and further details on the calculation can be found in [5]. 
An integration of (2) over the angles of the photon with index 2 leads to the one-photon distribution

$$
\frac{d^{2} \sigma_{\mathrm{DR}}}{d \omega d \Omega}(\omega ; \theta, \phi)=\int d \Omega^{\prime} \frac{d^{3} \sigma_{\mathrm{DR}}}{d \omega d \Omega d \Omega^{\prime}}\left(\omega ; \theta, \phi, \theta^{\prime}, \phi^{\prime}\right),
$$

which is of interest when only one of the photons is detected. A further calculation shows that

$$
\frac{d^{2} \sigma_{\mathrm{DR}}}{d \omega d \Omega}(\omega ; \theta, \phi)=\sum_{\substack{j, k=1 \\ j \neq k}}^{2} \sum_{d_{1}, d_{2}} \frac{d \sigma_{\mathrm{DR}}^{j, k}}{d \omega}\left(\omega ; d_{1}, d_{2}\right) W_{d_{1}, d_{2}}^{j, k}(\theta, \phi)
$$

where

$$
W_{d_{1}, d_{2}}^{1,2}(\theta, \phi) \equiv W_{d_{1}, d_{2}}^{1,2}(\theta)=\frac{1}{4 \pi} \sum_{\nu} \sqrt{2 \nu+1} \beta_{(\nu, 0) \nu}^{d_{1}, d_{2}, 1,2} P_{\nu}(\cos \theta)
$$

and

$$
\begin{aligned}
W_{d_{1}, d_{2}}^{2,1}(\theta, \phi) & \equiv W_{d_{1}, d_{2}}^{2,1}(\theta) \\
& =\frac{1}{4 \pi} \sum_{\nu} \sqrt{2 \nu+1} \beta_{(\nu, 0) \nu}^{d_{1}, d_{2}, 2,1} P_{\nu}(\cos \theta) \\
& =\frac{1}{4 \pi} \sum_{\nu} \sqrt{2 \nu+1} \beta_{(0, \nu) \nu}^{d_{1}, d_{2}, 1,2} P_{\nu}(\cos \theta)
\end{aligned}
$$

In the last step in (14), the symmetry relation

$$
\beta_{\left(\nu_{1}, \nu_{2}\right) \nu}^{d_{1}, d_{2}, 1,2}=\beta_{\left(\nu_{2}, \nu_{1}\right) \nu}^{d_{1}, d_{2}, 2,1}
$$

has been used. $P_{\nu}$ are the Legendre polynomials of degree $\nu$. We remark that (12) is still invariant under the transformation $\omega \mapsto E-E_{f}-\omega$, and that the total cross section is obtained from (2) by performing the following integration:

$$
\sigma_{\mathrm{DR}}=\frac{1}{2} \int_{0}^{E-E_{f}} d \omega \int d \Omega \frac{d^{2} \sigma_{\mathrm{DR}}}{d \omega d \Omega}(\omega ; \theta, \phi) .
$$

\section{B. Application to the RTE process}

The electrons bound in the target molecule may be regarded as quasi-free, and the impulse approximation is adopted [3,6]. Within this approximation, the effect of binding of electrons in the light target just gives rise to a momentum spread of the captured electron. In order to obtain the cross section for RTE, the DR cross section is convoluted with the electron momentum distribution in the target as seen from the projectile frame:

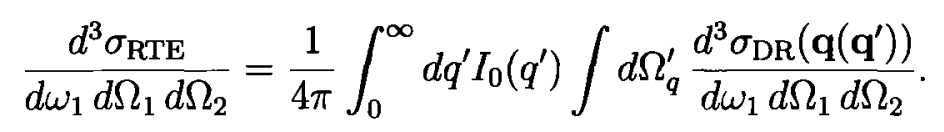


The integration is performed over the electron momentum $\mathbf{q}^{\prime}$ in the target frame, whereas $\mathbf{q}$ denotes the coordinates in the projectile frame. The distribution function $I_{0}\left(q^{\prime}\right)$ is an average over the direction of the molecule's symmetry axis and is thus spherically symmetric [7]. It is normalized to unity:

$$
\int d^{3} q^{\prime} \frac{I_{0}\left(q^{\prime}\right)}{4 \pi q^{2}}=\int_{0}^{\infty} d q^{\prime} I_{0}\left(q^{\prime}\right)=1
$$

Since we are only interested in the one-photon distribution here, we need the expression

$$
\frac{d^{2} \sigma_{\mathrm{RTE}}}{d \omega d \Omega}=\frac{1}{4 \pi} \int_{0}^{\infty} d q^{\prime} I_{0}\left(q^{\prime}\right) \int d \Omega^{\prime} \frac{d^{2} \sigma_{\mathrm{DR}}\left(\mathbf{q}\left(\mathbf{q}^{\prime}\right)\right)}{d \omega d \Omega}
$$

instead of (17). Note that $\omega$ stands for the photon energy in the projectile frame. The angular-differential cross section for electron capture with emission of a certain spectral line - as for example the $K \alpha_{1}^{\mathrm{S}}$ line - is computed by integrating (19) over the energy of the photon and summing over the set of pairs $\left(d_{1}, d_{2}\right)$ of states, which contribute to this line. We denote this set by $\mathcal{D}$. The main contributing states are listed in table I.

In the specific case of $K L L$-RTE from a hydrogen gas target into hydrogen-like uranium ions, the width of the Compton profile is relatively large compared to the widths $\Gamma$ and $A_{r}$ of the atomic states appearing in (6). We therefore use delta functions instead of Lorentz profiles in this expression and approximate the angular-differential cross section for RTE by

$$
\left(\frac{d \sigma_{\mathrm{RTE}}}{d \Omega}\right)_{\mathcal{D}}(\theta)=\frac{1}{\gamma v} \sum_{\substack{j, k=1 \\ j \neq k}}^{2} \sum_{\substack{\left.d_{1}, d_{2}\right) \in \mathcal{D} \\ W_{d_{1}, d_{2}}}}^{j, k}(\theta) R_{d_{1}, d_{2}}^{j, k} \mathcal{J}\left(q_{z}^{d_{1}}\right)
$$

The quantities $R_{d_{1}, d_{2}}^{j, k}$ describe the strengths of the resonances (see eq. (26)). The Compton profile $\mathcal{J}\left(q_{z}\right)$ gives the probability density to find a target electron with the momentum component $q_{z}$ in the projectile frame. It may be calculated from $I_{0}\left(q^{\prime}\right)$ by [7]

$$
\mathcal{J}\left(q_{z}\right)=\int d q_{x} d q_{y} \frac{I_{0}\left(q^{\prime}(\mathbf{q})\right)}{4 \pi q^{\prime}(\mathbf{q})^{2}}=\frac{1}{2} \int_{\left|q_{z}^{\prime}\left(q_{z}\right)\right|}^{\infty} d q^{\prime} \frac{I_{0}\left(q^{\prime}\right)}{q^{\prime}} .
$$

We obtained the Compton profile for the $\mathrm{H}_{2}$ target by interpolating values tabulated by Jeziorski et al. [8]. The momentum $q_{z}^{d_{1}}$ is found from the resonance energy $E_{d_{1}}$ by a Lorentz transformation $[3,6]$ :

$$
q_{z}^{d_{1}}=\frac{\gamma\left(c^{2}-\left|E_{\mathrm{bind}}\right|\right)-\left(E_{d_{1}}-E_{i}+c^{2}\right)}{\gamma v}
$$

where $E_{\mathrm{bind}}$ is the binding energy of the electron in the target and $E_{i}$ is the ground state energy of the inital hydrogen-like ion. 
TABLE I: Intermediate states $d_{1}$ and $d_{2}$, their energies $E_{d_{1}}$ and $E_{d_{2}}$, labelling of the photons, and resonance strengths $R_{d_{1}, d_{2}}$ for the most dominant cascades. (The dashes indicate that the emitted radiation belongs neither to the $K \alpha_{1}^{\mathrm{HS}}$ nor the $K \alpha_{2}^{\mathrm{HS}}$ energy range.)

\begin{tabular}{|c|c|c|c|c|c|c|c|c|}
\hline \begin{tabular}{|c|}
$\begin{array}{c}\text { Resonance } \\
\text { group }\end{array}$ \\
\end{tabular} & State $d_{1}$ & $\begin{array}{l}E_{d_{1}} \\
(\mathrm{eV})\end{array}$ & $\begin{array}{c}\text { first } \\
\text { photon }\end{array}$ & State $d_{2}$ & $\begin{array}{l}E_{d_{2}} \\
(\mathrm{eV})\end{array}$ & $\begin{array}{l}\text { second } \\
\text { photon }\end{array}$ & $\begin{array}{l}R_{d_{1}, d_{2}} \\
(\mathrm{~b} \mathrm{eV})\end{array}$ & $\begin{array}{c}\sum_{d_{2}} R_{d_{1}, d_{2}} \\
\quad(\mathrm{~b} \mathrm{eV})\end{array}$ \\
\hline \multirow{12}{*}{$K L_{1 / 2} L_{1 / 2}$} & \multirow{2}{*}[2s_{1/2}2p_{1/2}]{$_{0}$} & \multirow{2}{*}{-67896} & $K \alpha_{2}^{\mathrm{HS}}$ & {$\left[1 s_{1 / 2} 2 s_{1 / 2}\right]_{1}$} & -165369 & $K \alpha_{2}^{\mathrm{S}}$ & 18142 & \multirow{2}{*}{18215} \\
\hline & & & $K \alpha_{2}^{\mathrm{HS}}$ & {$\left[1 s_{1 / 2} 2 p_{1 / 2}\right]_{1}$} & -165225 & $K \alpha_{2}^{\mathrm{S}}$ & 72.9 & \\
\hline & \multirow{3}{*}[2s_{1/2}2s_{1/2}]{$_{0}$} & \multirow{3}{*}{-67833} & $K \alpha_{2}^{\mathrm{HS}}$ & {$\left[1 s_{1 / 2} 2 s_{1 / 2}\right]_{1}$} & -165369 & $K \alpha_{2}^{\mathrm{S}}$ & 54.9 & \multirow{3}{*}{15924} \\
\hline & & & $K \alpha_{2}^{\mathrm{HS}}$ & {$\left[1 s_{1 / 2} 2 p_{1 / 2}\right]_{1}$} & -165225 & $K \alpha_{2}^{\mathrm{S}}$ & 15868 & \\
\hline & & & - & {$\left[1 s_{1 / 2} 2 p_{3 / 2}\right]_{1}$} & -160783 & $K \alpha_{1}^{S}$ & 1.03 & \\
\hline & \multirow{4}{*}[2s_{1/2}2p_{1/2}]{$_{1}$} & \multirow{4}{*}{-67856} & $K \alpha_{2}^{\mathrm{HS}}$ & {$\left[1 s_{1 / 2} 2 s_{1 / 2}\right]_{1}$} & -165369 & $K \alpha_{2}^{\mathrm{S}}$ & 14158 & \multirow{4}{*}{20841} \\
\hline & & & $K \alpha_{2}^{\mathrm{HS}}$ & {$\left[1 s_{1 / 2} 2 p_{1 / 2}\right]_{1}$} & -165225 & $K \alpha_{2}^{\mathrm{S}}$ & 55.9 & \\
\hline & & & $K \alpha_{2}^{\mathrm{HS}}$ & {$\left[1 s_{1 / 2} 2 p_{1 / 2}\right]_{0}$} & -165114 & $K \alpha_{2}^{\mathrm{S}}$ & 27.9 & \\
\hline & & & $K \alpha_{2}^{\mathrm{HS}}$ & {$\left[1 s_{1 / 2} 2 s_{1 / 2}\right]_{0}$} & -165113 & $K \alpha_{2}^{\mathrm{S}}$ & 6599 & \\
\hline & \multirow{3}{*}[2p_{1/2}2p_{1/2}]{$_{0}$} & \multirow{3}{*}{-67712} & $K \alpha_{2}^{\mathrm{HS}}$ & {$\left[1 s_{1 / 2} 2 s_{1 / 2}\right]_{1}$} & -165369 & $K \alpha_{2}^{\mathrm{S}}$ & 32.8 & \multirow{3}{*}{6937} \\
\hline & & & $K \alpha_{2}^{\mathrm{HS}}$ & {$\left[1 s_{1 / 2} 2 p_{1 / 2}\right]_{1}$} & -165225 & $K \alpha_{2}^{\mathrm{S}}$ & 6902 & \\
\hline & & & 一 & {$\left[1 s_{1 / 2} 2 p_{3 / 2}\right]_{1}$} & -160783 & $K \alpha_{1}^{\mathrm{S}}$ & 2.20 & \\
\hline \multirow{16}{*}{$K L_{1 / 2} L_{3 / 2}$} & \multirow{4}{*}[2s_{1/2}2p_{3/2}]{$_{2}$} & \multirow{4}{*}{-63393} & $K \alpha_{1}^{\mathrm{HS}}$ & {$\left[1 s_{1 / 2} 2 s_{1 / 2}\right]_{1}$} & -165369 & $K \alpha_{2}^{\mathrm{S}}$ & 5462 & \multirow{4}{*}{5504} \\
\hline & & & $K \alpha_{1}^{\mathrm{HS}}$ & {$\left[1 s_{1 / 2} 2 s_{1 / 2}\right]_{0}$} & -165113 & $K \alpha_{2}^{\mathrm{S}}$ & 15.1 & \\
\hline & & & $K \alpha_{2}^{H S}$ & {$\left[1 s_{1 / 2} 2 p_{3 / 2}\right]_{2}$} & -160857 & $K \alpha_{1}^{\mathrm{S}}$ & 13.3 & \\
\hline & & & $K \alpha_{2}^{\mathrm{HS}}$ & {$\left[1 s_{1 / 2} 2 p_{3 / 2}\right]_{1}$} & -160783 & $K \alpha_{1}^{\mathrm{S}}$ & 13.3 & \\
\hline & \multirow{4}{*}[2p_{1/2}2p_{3/2}]{$_{1}$} & \multirow{4}{*}{-63355} & $K \alpha_{1}^{\mathbf{H S}}$ & {$\left[1 s_{1 / 2} 2 p_{1 / 2}\right]_{1}$} & -165225 & $K \alpha_{2}^{\mathrm{S}}$ & 334 & \multirow{4}{*}{2164} \\
\hline & & & $K \alpha_{1}^{\mathrm{HS}}$ & {$\left[1 s_{1 / 2} 2 p_{1 / 2}\right]_{0}$} & -165114 & $K \alpha_{2}^{\mathrm{S}}$ & 651 & \\
\hline & & & $K \alpha_{2}^{\mathrm{HS}}$ & {$\left[1 s_{1 / 2} 2 p_{3 / 2}\right]_{2}$} & -160857 & $K \alpha_{1}^{\mathrm{S}}$ & 984 & \\
\hline & & & $K \alpha_{2}^{\mathrm{HS}}$ & {$\left[1 s_{1 / 2} 2 p_{3 / 2}\right]_{1}$} & -160783 & $K \alpha_{1}^{\mathrm{S}}$ & 195 & \\
\hline & \multirow{4}{*}[2p_{1/2}2p_{3/2}]{$_{2}$} & \multirow{4}{*}{-63339} & $K \alpha_{1}^{\mathrm{HS}}$ & {$\left[1 s_{1 / 2} 2 p_{1 / 2}\right]_{1}$} & -165225 & $K \alpha_{2}^{\mathrm{S}}$ & 8579 & \multirow{4}{*}{19004} \\
\hline & & & $K \alpha_{1}^{\mathrm{HS}}$ & {$\left[1 s_{1 / 2} 2 p_{1 / 2}\right]_{0}$} & -165114 & $K \alpha_{2}^{S}$ & 24.0 & \\
\hline & & & $K \alpha_{2}^{\mathrm{HS}}$ & {$\left[1 s_{1 / 2} 2 p_{3 / 2}\right]_{2}$} & -160857 & $K \alpha_{1}^{\mathrm{S}}$ & 5266 & \\
\hline & & & $K \alpha_{2}^{\mathrm{HS}}$ & {$\left[1 s_{1 / 2} 2 p_{3 / 2}\right]_{1}$} & -160783 & $K \alpha_{1}^{\mathrm{S}}$ & 5135 & \\
\hline & \multirow{4}{*}[2s_{1/2}2p_{3/2}]{$_{1}$} & \multirow{4}{*}{-63268} & $K \alpha_{1}^{\mathrm{HS}}$ & {$\left[1 s_{1 / 2} 2 s_{1 / 2}\right]_{1}$} & -165369 & $K \alpha_{2}^{\mathrm{S}}$ & 2671 & \multirow{4}{*}{8384} \\
\hline & & & $K \alpha_{1}^{\mathrm{HS}}$ & {$\left[1 s_{1 / 2} 2 s_{1 / 2}\right]_{0}$} & -165113 & $K \alpha_{2}^{\mathrm{S}}$ & 5672 & \\
\hline & & & $K \alpha_{2}^{\mathrm{HS}}$ & {$\left[1 s_{1 / 2} 2 p_{3 / 2}\right]_{2}$} & -160857 & $K \alpha_{1}^{\mathrm{S}}$ & 33.8 & \\
\hline & & & $K \alpha_{2}^{\mathrm{HS}}$ & {$\left[1 s_{1 / 2} 2 p_{3 / 2}\right]_{1}$} & -160783 & $K \alpha_{1}^{\mathrm{S}}$ & 6.75 & \\
\hline \multirow{4}{*}{$K L_{3 / 2} L_{3 / 2}$} & \multirow{2}{*}[2p_{3/2}2p_{3/2}]{$_{2} \mid$} & $\mid-58828$ & $K \alpha_{1}^{\mathrm{HS}}$ & {$\left[1 s_{1 / 2} 2 p_{3 / 2}\right]_{2}$} & -160857 & $K \alpha_{1}^{\mathrm{S}}$ & 3650 & \\
\hline & & -58828 & $K \alpha_{1}^{\mathrm{HS}}$ & {$\left[1 s_{1 / 2} 2 p_{3 / 2}\right]_{1}$} & -160783 & $K \alpha_{1}^{\mathrm{S}}$ & 3701 & 7351 \\
\hline & 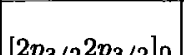 & $-58733 \mid$ & $K \alpha_{1}^{\mathrm{HS}}$ & {$\left[1 s_{1 / 2} 2 p_{3 / 2}\right]_{2}$} & -160857 & $K \alpha_{1}^{\mathrm{S}}$ & 9.10 & 1302 \\
\hline & {$\left[2 p_{3 / 2} 2 p_{3 / 2}\right] 0$} & & $K \alpha_{1}^{H S}$ & {$\left[1 s_{1 / 2} 2 p_{3 / 2}\right]_{1}$} & -160783 & $K \alpha_{1}^{\mathrm{S}}$ & 1293 & \\
\hline
\end{tabular}

If the natural line widths for the different lines are smaller than their energy separation, it is possible to distinguish between the two photons. This means that the photon with index 1 can indeed be regarded as the first photon (the hypersatellite). The angular-differential cross 
section is then found to be

$$
\left(\frac{d \sigma_{\mathrm{RTE}}}{d \Omega}\right)_{\mathcal{D}, i}(\theta)=\frac{1}{4 \pi} \sigma_{\mathcal{D}, i}^{\mathrm{RTE}} \sum_{\nu} \beta_{\mathcal{D}, i}^{\mathrm{eff}, \nu} P_{\nu}(\cos \theta),
$$

where $i$ denotes the number of the photon which is detected.

The partial cross sections appearing in (23) are given by

$$
\sigma_{\mathcal{D}, 1}^{\mathrm{RTE}}=\frac{1}{\gamma v} \sum_{\left(d_{1}, d_{2}\right) \in \mathcal{D}} R_{d_{1}, d_{2}} \mathcal{J}\left(q_{z}^{d_{1}}\right)
$$

for the first photon and

$$
\sigma_{\mathcal{D}, 2}^{\mathrm{RTE}}=\frac{1}{\gamma v} \sum_{\left(d_{1}, d_{2}\right) \in \mathcal{D}} \frac{A_{\tau}\left(d_{2}, f\right)}{\Gamma_{d_{2}}} R_{d_{1}, d_{2}} \mathcal{J}\left(q_{z}^{d_{1}}\right)
$$

for the second photon. The resonance strengths are

$$
R_{d_{1}, d_{2}}:=R_{d_{1}, d_{2}}^{1,2}=\frac{2 \pi^{2}}{p^{2}} \frac{A_{r}\left(d_{1}, d_{2}\right)}{\Gamma_{d_{1}}} V_{a}\left(d_{1}\right),
$$

and the effective anisotropy parameters in (23) are calculated by

$$
\beta_{\mathcal{D}, 1}^{\text {eff }, \nu}=\sqrt{2 \nu+1}\left(\sum_{\left(d_{1}, d_{2}\right) \in \mathcal{D}} R_{d_{1}, d_{2}} \mathcal{J}\left(q_{z}^{d_{1}}\right)\right)^{-1} \sum_{\left(d_{1}, d_{2}\right) \in \mathcal{D}} R_{d_{1}, d_{2}} \mathcal{J}\left(q_{z}^{d_{1}}\right) \beta_{(\nu, 0) \nu}^{d_{1}, d_{2}, 1,2}
$$

and

$$
\begin{aligned}
\beta_{\mathcal{D}, 2}^{\mathrm{eff}, \nu}= & \sqrt{2 \nu+1}\left(\sum_{\left(d_{1}, d_{2}\right) \in \mathcal{D}} \frac{A_{r}\left(d_{2}, f\right)}{\Gamma_{d_{2}}} R_{d_{1}, d_{2}} \mathcal{J}\left(q_{z}^{d_{1}}\right)\right)^{-1} \\
& \times \sum_{\left(d_{1}, d_{2}\right) \in \mathcal{D}} \frac{A_{r}\left(d_{2}, f\right)}{\Gamma_{d_{2}}} R_{d_{1}, d_{2}} \mathcal{J}\left(q_{z}^{d_{1}}\right) \beta_{(0, \nu) \nu}^{d_{1}, d_{2}, 1,2}
\end{aligned}
$$

\section{NUMERICAL RESULTS AND DISCUSSION}

The relativistic resonance energies, bound state wavefunctions, and the radiative transition rates are obtained with the GRASP multi-configurational Dirac-Fock atomic structure code by Dyall et al. [9]. Nuclear finite size effects are taken into account by using a twoparameter Fermi charge distribution. Lowest-order QED corrections are included in the energies in an approximate manner. 
TABLE II: Partial cross sections $\sigma_{\mathcal{D}, 1}^{\mathrm{RTE}}$ for the first photon (hypersatellite, HS) and $\sigma_{\mathcal{D}, 2}^{\mathrm{RTE}}$ for the second photon (satellite, S) in barn.

Projectile energy

\begin{tabular}{clccc}
$(\mathrm{MeV} / \mathrm{u})$ & $K \alpha_{1}^{\mathrm{HS}}$ & $K \alpha_{2}^{\mathrm{HS}}$ & $K \alpha_{1}^{\mathrm{S}}$ & $K \alpha_{2}^{\mathrm{S}}$ \\
\hline 116.6 & 0.0299 & 21.7 & 0.0141 & 21.7 \\
124.9 & 7.94 & 4.22 & 3.35 & 8.81 \\
133.1 & 2.90 & 0.0644 & 2.48 & 0.481 \\
\hline \hline
\end{tabular}

A partial-wave expansion is used for the wavefunction of the incoming electron with asymptotic momentum $\mathbf{p}$ and spin projection $m_{s}$ :

$$
\psi_{\mathbf{p} m_{s}}(\mathbf{r})=\sum_{\kappa \mu} i^{l} e^{i \Delta_{\kappa}} \sum_{m_{l}} Y_{l}^{m_{l} *}(\hat{\mathbf{p}}) C\left(l \frac{1}{2} j ; m_{l} m_{s} \mu\right) \psi_{\mathbf{p} \kappa}(\mathbf{r})
$$

The partial-wave components $\psi_{p \kappa \mu}(\mathbf{r})$ are calculated numerically by integrating the Dirac equation with the nuclear potential screened by the bound $1 s$ electron. This task and the evaluation of matrix elements for the capture of the continuum electron are performed by the Auger code of Zimmerer [10].

The phases $\Delta_{\kappa}$ in (29) ensure that the wavefunction satisfies the boundary condition of an incoming plane wave and an outgoing spherical wave. They are determined by matching the numerical solution integrated in the interior region with analytic Dirac-Coulomb functions of the exterior region.

The theory developed above will now be applied to a comparison with the experiment performed by Ma et al. [1], who observed the x-ray emission after collisions of $U^{91+}$ ions with a hydrogen gas target. We consider here intensity ratios since they are experimentally accessible with a larger precision than absolute cross sections.

Partial RTE cross sections for $K \alpha_{1}\left(2 p_{3 / 2} \rightarrow 1 s_{1 / 2}\right)$ and $K \alpha_{2}\left(2 s_{1 / 2}, 2 p_{1 / 2} \rightarrow 1 s_{1 / 2}\right)$ radiation as calculated with (24) are shown in Fig. 1. Within the resonance groups, the single DR resonances listed in table I cannot be resolved since they are broadened by the target Compton profile. The values of the partial cross sections at the experimental energies are displayed in table II and are compared to the measurement in table 2 of the companion paper [1]. Note that the cross sections are understood with respect to one target electron.

Figure 2 shows the effective dipole anisotropy parameters $\beta^{\text {eff, } \nu=2}$ introduced in (27) and 


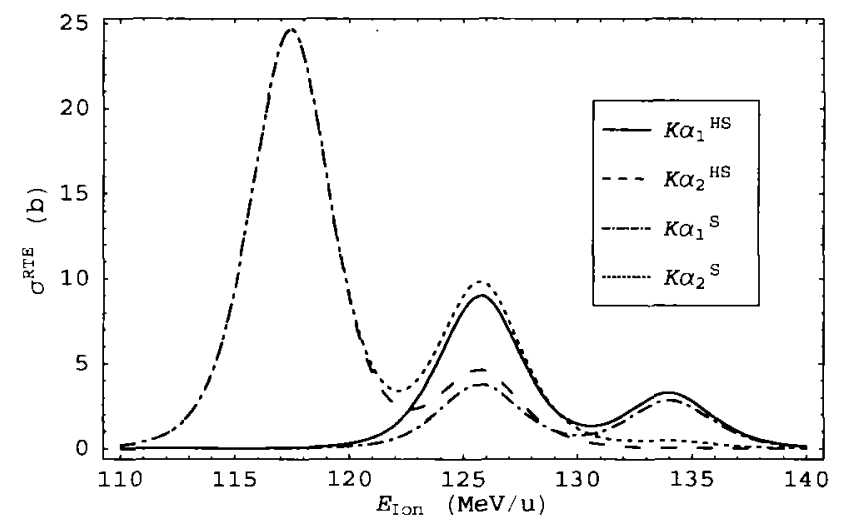

FIG. 1: Partial RTE cross sections for emission of $K \alpha_{1}$ and $K \alpha_{2}$ radiation in the range of the $K L L$ resonances as function of uranium lab energy.

TABLE III: Effective dipole anisotropy parameters $\beta_{\mathcal{D}, 1}^{\text {eff,2 }}$ for the first photon (hypersatellite, HS) and $\beta_{\mathcal{D}, 2}^{\text {eff } 2}$ for the second photon (satellite, S).

Projectile energy

\begin{tabular}{ccccc}
$(\mathrm{MeV} / \mathrm{u})$ & $K \alpha_{1}^{\mathrm{HS}}$ & $K \alpha_{2}^{\mathrm{HS}}$ & $K \alpha_{1}^{\mathrm{S}}$ & $K \alpha_{2}^{\mathrm{S}}$ \\
\hline 116.6 & -0.374 & $-2.02 \times 10^{-3}$ & -0.239 & -0.0286 \\
124.9 & -0.384 & $5.18 \times 10^{-3}$ & -0.260 & -0.206 \\
133.1 & -0.0177 & $4.58 \times 10^{-3}$ & -0.230 & -0.493 \\
\hline
\end{tabular}

(28) selectively for $K \alpha_{1}$ and $K \alpha_{2}$ emission in the energy range of $K L L$ transitions. Note that only dipole radiation was regarded in these calculations, in which case (23) becomes

$$
\left(\frac{d \sigma_{\mathrm{RTE}}}{d \Omega}\right)_{\mathcal{D}, i}(\theta)=\frac{1}{4 \pi} \sigma_{\mathcal{D}, i}^{\mathrm{RTE}}\left(1+\beta_{\mathcal{D}, i}^{\mathrm{eff}, 2} P_{2}(\cos \theta)\right)=\frac{1}{4 \pi} \sigma_{\mathcal{D}, i}^{\mathrm{RTE}}\left(1+\frac{1}{2} \beta_{\mathcal{D}, i}^{\mathrm{eff}, 2}\left(3 \cos ^{2} \theta-1\right)\right) .
$$

For the cascades via the singly-excited $\left[1 s_{1 / 2} 2 p_{3 / 2}\right]_{2}$ state, which decays first into the state $\left[1 s_{1 / 2} 2 s_{1 / 2}\right]_{1}$ with a branching ratio of around $30 \%$, the third photon is the satellite. The anisotropy of this photon is also incorporated in the numerical results. The values of the effective anisotropy parameters at the experimental energies are found in table III. At a projectile energy of $116.6 \mathrm{MeV}$, which is in the range of the $K L_{1 / 2} L_{1 / 2}$ resonance group, the $K \alpha_{1}^{\mathrm{HS}}$ emission is strongly anisotropic.

In Fig. 3, the ratio of the $K \alpha_{1}$ and $K \alpha_{2}$ HS intensities is plotted against the emission 


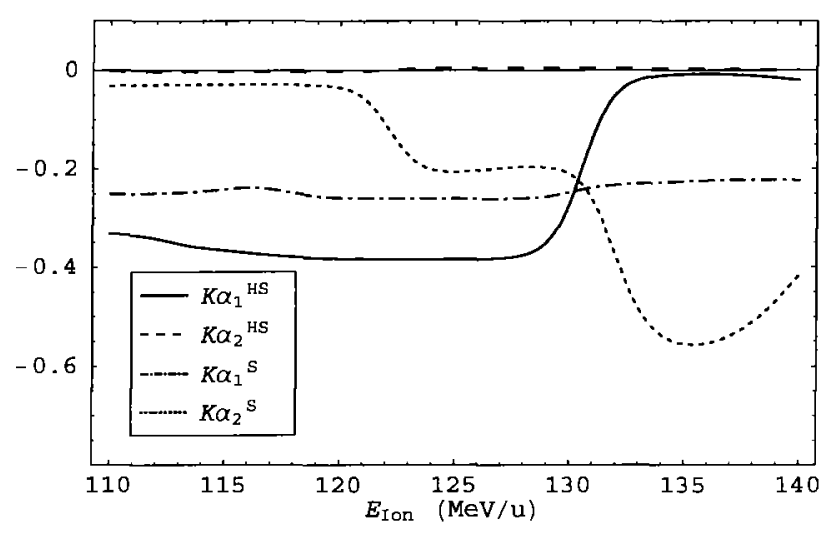

FIG. 2: Effective dipole anisotropy parameters $\beta_{\mathcal{D}, i}^{\text {eff,2 }}$ for HS $(i=1)$ and $\mathrm{S}(i=2)$ emission as function of the ion energy in the range of the $K L L$ resonances.

angle in the laboratory frame at $124.9 \mathrm{MeV} / \mathrm{u}$, which corresponds to $K L_{1 / 2} L_{3 / 2}$ resonances. Hypersatellite lines are not perturbed by the REC contribution since the doubly-excited states from which they are emitted can only be populated by RTE. A comparison with Fig. 2 reveals that the $K \alpha_{2}^{\mathrm{HS}}$ line is isotropic. This is confirmed by the measurements (see Fig. 8 and the related discussion in [1]). Therefore the anisotropy of the intensity ratio stems from the $K \alpha_{1}^{\mathrm{HS}}$ radiation. The theoretical curve is in qualitative agreement with the experimental data from [1]. However, our calculation underestimates the intensity ratios at $60^{\circ}$ and $90^{\circ}$. This deviation might be removed by taking into account interferences between different multipole orders of the radiation. Surzhykov et al. [11] showed how the interference between $\mathrm{E} 1$ and M2 transitions in the $K \alpha_{1}$ decay in hydrogen-like heavy ions may modify the angular distributions of the emitted photons when an alignment in the $L$-shell is present. The RTE process also produces aligned states.

Figures 4 and 5 show the angular distribution of the $K \alpha_{1}^{\mathrm{HS}}$ and $K \alpha_{2}^{\mathrm{HS}}$ radiation in the $K L_{1 / 2} L_{3 / 2}$ resonance group. The experimental data points have been normalized to the measured angular distribution of the $K \alpha_{2}^{\mathrm{S}}$ radiation, which has been proven experimentally to be isotropic for all the resonance groups, even at a non-resonant projectile energy (see Fig. 6 and explanations in [1]). Although we have found that the $K \alpha_{2}^{\mathrm{S}}$ emission of the RTE process possesses a strong angular dependence (see Fig. 2 and table III), the total $K \alpha_{2}^{\mathrm{S}}$ intensity is dominated by isotropically emitted photons following REC into the $L$-shell or higher shells. Therefore the $K \alpha_{2}^{\mathrm{S}}$ radiation can be regarded as isotropic at the level of experimental accuracy. In our calculation we adjusted the angular-independent cross section 


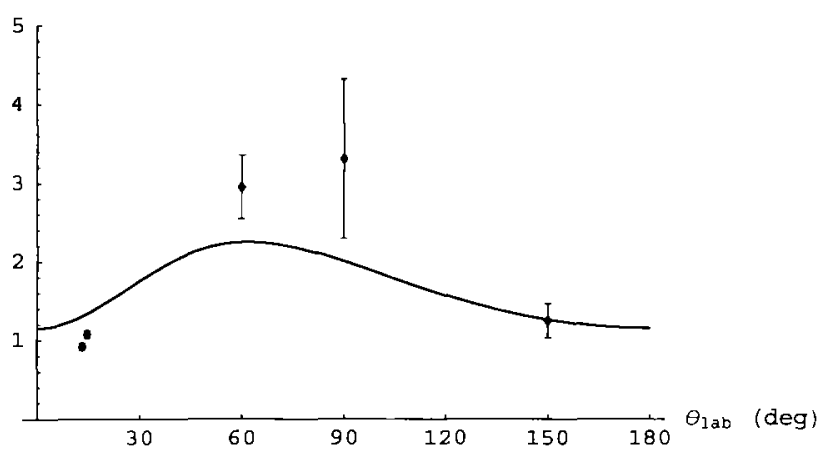

FIG. 3: Ratio of the $K \alpha_{1}^{\mathrm{HS}}$ and $K \alpha_{2}^{\mathrm{HS}}$ intensities at an ion energy of $124.9 \mathrm{MeV} / \mathrm{u}$. Experimental values were taken from $[1]$.

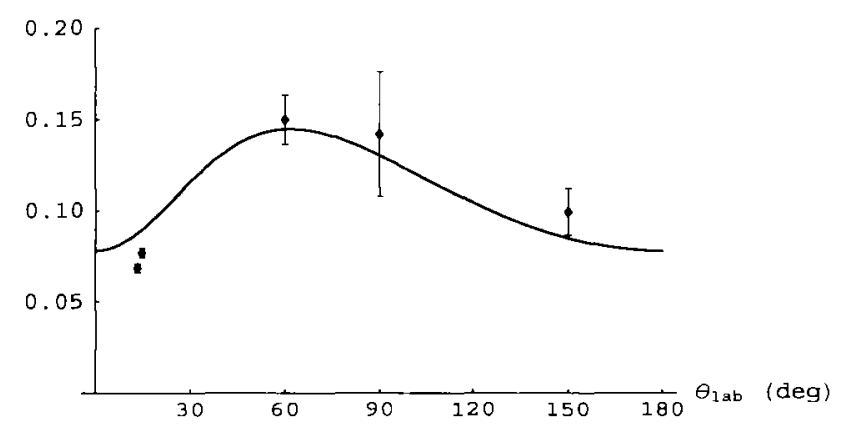

FIG. 4: Ratio of the $K \alpha_{1}^{\mathrm{HS}}$ and $K \alpha_{2}^{\mathrm{S}}$ intensities at an ion energy of $124.9 \mathrm{MeV} / \mathrm{u}$. Experimental values were taken from [1].

$\sigma_{K \alpha_{2}^{\mathrm{S}}}^{\mathrm{REC}}$ of the de-excitation $\mathrm{X}$ rays from REC in order to fit the experimental intensity ratios. Neglecting interferences between RTE and REC, we have

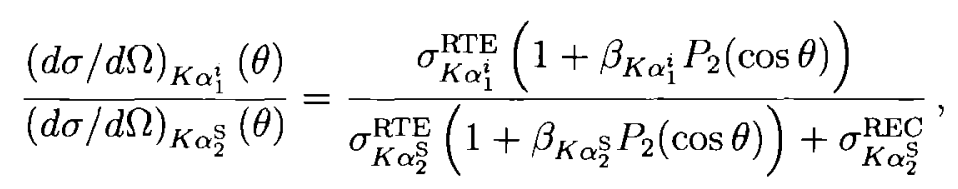

where $i$ stand for $\mathrm{HS}$ or $\mathrm{S}$, and the value $\sigma_{K \alpha_{2}^{\mathrm{S}}}^{\mathrm{REC}}=55.6 \mathrm{~b}$ is found. So this analysis also yields an estimate for the REC cross section, showing that it indeed overweighs the RTE cross section of $17.6 \mathrm{~b}$. With the above value, we see a reasonable agreement between experiment and theory, in particular in Fig. 4.

Figure 6 contains the angular distribution of $K \alpha_{1}^{\mathrm{HS}}$ radiation emitted in the range of the $K L_{3 / 2} L_{3 / 2}$ group. Here we followed the same normalization procedure as explained for the previous figures and found the REC cross section $\sigma_{K \alpha_{2}^{\mathrm{S}}}^{\mathrm{REC}}=74.6 \mathrm{~b}$. Comparing this to the RTE contribution of only $0.9 \mathrm{~b}$, we can conclude that the REC process is even more 


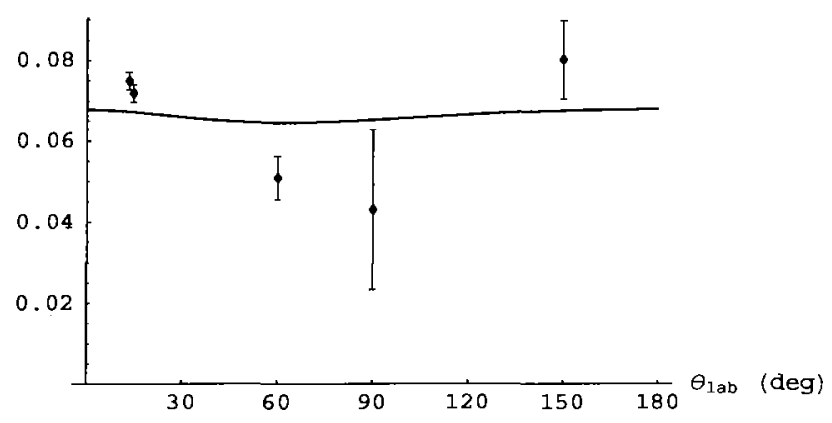

FIG. 5: Ratio of the $K \alpha_{2}^{\mathrm{HS}}$ and $K \alpha_{2}^{\mathrm{S}}$ intensities at an ion energy of $124.9 \mathrm{MeV} / \mathrm{u}$. Experimental values were taken from [1].

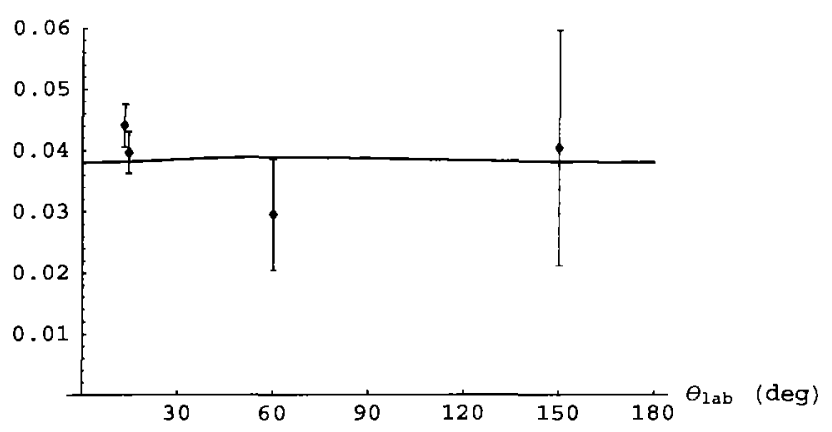

FIG. 6: Ratio of the $K \alpha_{1}^{\mathrm{HS}}$ and $K \alpha_{2}^{\mathrm{S}}$ intensities at an ion energy of $133.1 \mathrm{MeV} / \mathrm{u}$. Experimental values were taken from [1].

dominant for the third resonance group. The agreement is again satisfactory within the experimental errors.

\section{SUMMARY}

In this paper we studied the angular distribution of radiation emitted after $K L L$-RTE into $\mathrm{U}^{91+}$ ions. The dipole coefficients for the radiation appearing in the expansion in coupled spherical harmonics were calculated explicitly. From this expansion, both hypersatellite and satellite angular distributions were obtained. We applied the impulse approximation to compute differential cross sections for RTE in collisions of uranium ions with a hydrogenmolecule gas target. The comparision of our results to experimental data shows a good qualitative agreement. A future extension of this work should also account for interference effects with higher multipole orders of the radiation. 
The studies presented here give detailed information about the alignment of intermediate states after the $K L L$-RTE capture into $\mathrm{U}^{91+}$ and, therefore, lead to a deeper understanding of the electron-electron interaction in the dynamics of the capture process. In this context, we point out the importance of the Breit interaction for a capture in the $K L_{1 / 2} L_{1 / 2}$ resonance group. We note that our calculations also help to analyze the experimental spectra by examining the angular distribution of the radiation.

In summary, these investigations provide a clear interpretation of the capture process and the following photon emissions.

\section{Acknowledgments}

The authors would like to thank the experimental colleagues Prof. P. Mokler at GSI Darmstadt and Prof. X. Ma at IMP Lanzhou for stimulating discussions.

[1] X. Ma et al., Phys. Rev. A (joint to this issue).

[2] Th. Kandler et al., Phys. Lett. A 204, 274 (1995).

[3] M. Gail, N. Grün, and W. Scheid, J. Phys. B 31, 4645 (1998).

[4] V. V. Balashov, I. V. Bodrenko, V. K. Dolinov, and S. I. Strakhova, Opt. Spectrosc. (USSR) 77, 801 (1994).

[5] S. Zakowicz, Diploma thesis, University of Giessen, 2001; S. Zakowicz, W. Scheid, and N. Grün, Nucl. Instrum. Methods B 205, 386 (2003).

[6] J. Eichler and W. E. Meyerhof, Relativistic Atomic Collisions (Academic Press, San Diego, $1995)$.

[7] R. E. Brown and V. H. Smith, Phys. Rev. A 5, 140 (1972).

[8] B. Jeziorski and K. Szalewicz, Phys. Rev. A 19, 2360 (1979).

[9] K. G. Dyall, I. P. Grant, C. T. Johnson, F. A. Parpia, and E. P. Plummer, Comput. Phys. Commun. 55, 425 (1989).

[10] P. Zimmerer, N. Grün, and W. Scheid, Phys. Lett. A 148, 457 (1990).

[11] A. Surzhykov, S. Fritzsche, A. Gumberidze, and Th. Stöhlker, Phys. Rev. Lett. 88, 153001 (2002). 